\title{
Instantons on hyperkähler manifolds
}

\author{
Chandrashekar Devchand $^{1}$ (D) $\cdot$ Massimiliano Pontecorvo $^{2}$ (D) $\cdot$ Andrea Spiro $^{3}$
}

Received: 18 December 2018 / Accepted: 6 June 2019 / Published online: 22 July 2019

(c) The Author(s) 2019

\begin{abstract}
An instanton $(E, D)$ on a (pseudo-)hyperkähler manifold $M$ is a vector bundle $E$ associated with a principal $G$-bundle with a connection $D$ whose curvature is pointwise invariant under the quaternionic structures of $T_{x} M, x \in M$, and thus satisfies the Yang-Mills equations. Revisiting a construction of solutions, we prove a local bijection between gauge equivalence classes of instantons on $M$ and equivalence classes of certain holomorphic functions taking values in the Lie algebra of $G^{\mathbb{C}}$ defined on an appropriate $\mathrm{SL}_{2}(\mathbb{C})$-bundle over $M$. Our reformulation affords a streamlined proof of Uhlenbeck's compactness theorem for instantons on (pseudo-)hyperkähler manifolds.
\end{abstract}

Keywords Yang-Mills theory · Instantons $\cdot$ Hyperkähler geometry $\cdot$ Harmonic space Mathematics Subject Classification 70S15 $\cdot 14 \mathrm{D} 21 \cdot 53 \mathrm{C} 28 \cdot 53 \mathrm{C} 26 \cdot 32 \mathrm{~L} 05 \cdot 58 \mathrm{D} 27$

\section{Introduction}

Beginning in the mid-1970s, the self-duality equations for Yang-Mills fields successfully captured the imagination of theoretical physicists and mathematicians, epitomised by Don-

This research was partially supported by Ministero dell'Istruzione, Università e Ricerca in the framework of the project "Real and Complex Manifolds: Geometry, Topology and Harmonic Analysis" and by GNSAGA of INdAM.

$\bigotimes$ Chandrashekar Devchand devchand@aei.mpg.de

Massimiliano Pontecorvo max@mat.uniroma3.it

Andrea Spiro

andrea.spiro@unicam.it

1 Max-Planck-Institut für Gravitationsphysik (Albert-Einstein-Institut), Am Mühlenberg 1, 14476 Potsdam, Germany

2 Dipartimento di Matematica e Fisica, Università Roma Tre, Largo San Leonardo Murialdo 1, 00146 Rome, Italy

3 Scuola di Scienze e Tecnologie, Università di Camerino, Via Madonna delle Carceri, 62032 Camerino, Macerata, Italy 
aldson's flight into previously unforeseen realms of four-manifold differential topology (reviewed for instance in [21,29]). A Yang-Mills field is a pair $(E, D)$ on a Riemannian manifold $(M, g)$, where $E$ is a vector bundle associated with a principle $G$-bundle with a connection $D$ whose curvature satisfies the Yang-Mills equation $D * F=0$. The (anti-)self-duality equations, requiring that the curvature $F$ of a connection $D$ over a Riemannian four-manifold $(M, g)$ takes values in the (anti-)self-dual eigenspace of the Hodge star operator, implies the Yang-Mills equation in virtue of the Bianchi identity $D F=0$. Connections satisfying the (anti-)self-dual Yang-Mills (SDYM) equations are called (anti-) instantons. They are global minimisers of the Yang-Mills energy functional, $S(A)=\|F\|^{2}=\int_{M} F \wedge * F \operatorname{vol}_{g}$.

The quest for explicit instanton solutions [6] was initially physically motivated, for instance by the mystery of the phenomenon of quark confinement [33], but the remarkable properties of instantons soon attracted powerful mathematical treatment. First, Ward showed that solutions of the self-duality equations on $\mathbb{R}^{4}$ are encoded in certain holomorphic data on twistor space [40], effectively converting the problem to an algebro-geometric one. Then, Atiyah et al. [4] obtained a correspondence between solutions of the SDYM equations on $S^{4}$ and certain real algebraic bundles on the complex projective 3 -space $\mathbb{C} P^{3}$. They thus established the relation between self-duality and holomorphic structures, yielding in particular the dimension of the moduli space of solutions for any compact gauge group. This led to a sequence of ansätze yielding SDYM solutions in terms of arbitrary solutions of linear equations [5,10]. Subsequently, powerful algebro-geometric results were used to obtain a complete construction of all SDYM fields on $S^{4}[2,3,20]$.

These developments were followed by fundamental analytical results on variational methods for Yang-Mills theory. The moduli space of instantons is a subset of the quotient $\mathcal{A} / \mathcal{G}$ of the space of all connections $\mathcal{A}$ with the group of all gauge transformations $\mathcal{G}$. Locally representing the connections in Coulomb gauges Uhlenbeck $[37,38]$ developed analytical tools to study the singularities of the compact moduli space of instantons. Uhlenbeck's work, together with the novel variational methods introduced by Taubes to study gauge invariant theories, prepared the path for Donaldson's seminal work.

These analytical results depended crucially on the fact that the Yang-Mills functional and therefore also the Yang-Mills equations are conformally invariant in four dimensions. Further, the above-mentioned constructions of SDYM solutions crucially used the fact that $\mathbb{R}^{4}$ conformally compactifies to $S^{4}$. All this would seem to impede any generalisation to Yang-Mills fields in higher dimensions. Indeed, it is known that a connection over the sphere $S^{d}, d \geq 5$, with sufficiently small $L^{2}$-norm is necessarily flat [8]. However, the solvability of four-dimensional SDYM equations relies in particular on the fact that, being linear algebraic constraints on the curvature, they are first-order equations for the vector potential. This first-order property was partly responsible for the good analytical properties of the SDYM equations. Indeed, an insistence upon this familiar sight of partial-flatness conditions, requiring the vanishing of certain linear combinations of the curvature components, which automatically imply the second-order Yang-Mills equations, yields the required instanton equations in dimensions greater than four. This idea was originally pursued in [9], where it was shown that the required equations are restrictions of the curvature $F$ to an eigenspace of an endomorphism on the space of 2-forms defined by an appropriate co-closed 4-form $\Omega$,

$$
*(* \Omega \wedge F)=\lambda F, \quad \Omega \in \Lambda^{4} T^{*} M, \quad \lambda \in \mathbb{R}^{*} .
$$

The co-closedness of $\Omega$ suffices to show that a Yang-Mills curvature field satisfying (1.1) implies the second-order Yang-Mills equations. For $d>4$, the 4 -form $\Omega$ is pointwise 
invariant only under some proper subgroup of $\mathrm{SO}_{d}(\mathbb{R})$. The existence of $\Omega$ corresponds to some special holonomy on the manifold [1,17,19,25,36,39].

The above-mentioned compactness results for the moduli space of Yang-Mills fields were already generalised to higher dimensions by Uhlenbeck and Nakajima [30]. For the higher-dimensional generalisations of the self-duality equations, the analytical programme in the spirit of Uhlenbeck and Taubes was begun by Tian [36]. The investigation of (local) solutions of higher- dimensional equations of the form (1.1) began $[1,11,22,41]$ with the case of instantons on spaces having hyperkähler (hk) structure (i.e. with holonomy in $\mathrm{Sp}_{n}$ ), these being natural generalisations of $\mathbb{R}^{4}=\mathbb{H}$. Some global results on general instantons on quaternionic Kähler (qk) manifolds (with holonomy in $\mathrm{Sp}_{n} \cdot \mathrm{Sp}_{1}$ ) also exist; e.g. [28,31].

The twistor formulation of SDYM, which led to the ADHM construction, has a (local) field theory variant, the harmonic space formulation. This was originally developed as a tool to study supersymmetric harmonic maps $[22,24]$. The harmonic space formulation enlarges the $\mathbb{C} P^{1}$ fibre of the twistor bundle to $\mathrm{SL}_{2}(\mathbb{C})$, yielding a total space more amenable to standard field theoretical treatment. In the process, (gauge equivalent classes of) local solutions of selfdual theories are parametrised by a prepotential, much as the Kähler potential parametrises Kähler metrics. In a previous paper by two of us [16], we have given a differential geometric description of the corresponding construction of (pseudo-)hyperkähler metrics. In the current paper, we investigate properties of Yang-Mills instantons on (pseudo-)hyperkähler manifolds using the harmonic space formulation, presenting a differential geometric formulation of the method based on the work of [1].

The harmonic space of an hk manifold $(M, g)$ is the trivial bundle $\mathcal{H}(M)=\mathrm{SL}_{2}(\mathbb{C}) \times$ $M \rightarrow M$, equipped with a certain (non-product) complex structure. The space $\mathcal{H}(M)$ fibres naturally over the quotient $Z(M)=\mathrm{SL}_{2}(\mathbb{C}) / B \times M \simeq \mathbb{C} P^{1} \times M$, where $B$ is the Borel subgroup of upper triangular matrices. $Z(M)$ is the twistor bundle of $(M, g)$ and has a welldefined complex structure, canonically determined by the hypercomplex structure of $M$. Now, the complex structure of the harmonic space $\mathcal{H}(M)$ is the unique complex structure such that the projection $p: \mathcal{H}(M) \rightarrow Z(M)$ is holomorphic.

A gauge field $(E, D)$ on a complex hyperkähler manifold $(M, g)$ is an instanton if the curvature of $D$ is pointwise invariant under the quaternionic structure of $T_{p} M, p \in M$. Its pull-back field $\left(E^{\prime}, D^{\prime}\right)$ over $\mathcal{H}(M)$ admits an analytic gauge condition, by which we mean a special class of local trivialisations (= gauges) of $E^{\prime}$. This class has the crucial feature that its gauge transformations are holomorphic, supplemented by some other conditions. In such a trivialisation, the potential $A^{\prime}$ of $D^{\prime}$ is completely determined by just one of its components, which is moreover a holomorphic function on $\mathcal{H}(M)$. This component is called the prepotential of the gauge field $\left(E^{\prime}, D^{\prime}\right)$. A freely specifiable holomorphic prepotential, satisfying an appropriate first-order linear equation on $\mathcal{H}(M)$, encodes all local properties of the corresponding instanton solution on $M$ and may be used to reconstruct the associated Yang-Mills field $(E, D)$. This construction, together with complete proofs of an essentially bijective correspondence between normalised prepotentials on $\mathcal{H}(M)$ and moduli of locally defined instantons on $M$, takes up the bulk of the content of this paper.

The existence of the analytic gauge condition and the resulting holomorphic prepotential allows the use of classical results on holomorphic functions, such as Montel's theorem or Hartogs' removable singularity theorem, to investigate the moduli spaces of instantons. Thus, in this formulation, holomorphy provides very useful tools. This is analogous to Uhlenbeck's Coulomb gauge condition, which allows the use of the machinery of elliptic equations. As an example, we establish some simple estimates relating $\mathcal{C}^{k}$-norms of prepotentials to those of curvatures. These estimates, combined with the classical Montel theorem of complex variable 
theory, lead to a new direct proof of Uhlenbeck's strong compactness theorem for instantons on hk manifolds [18,30,36-38,42,43].

The paper is structured as follows. After the preliminary Sect. 2, we introduce the notion of harmonic space and discuss its relation with the twistor bundle of a (pseudo-)hyperkähler manifold $M$ in Sect. 3. In Sect. 4, we discuss the analytic gauge condition of the pull-back of the instanton over $\mathcal{H}(M)$ and the construction of the instanton field over $M$ from the corresponding holomorphic prepotential on $\mathcal{H}(M)$. Our main new contributions appear in Sects. 4 and 5 , where we introduce a convenient normalisation for equivalent prepotentials, prove a new existence result for an essentially unique instanton corresponding to a given prepotential, obtain curvature estimates and present our new brief proof of the strong compactness theorem for instantons on hk manifolds.

\section{Preliminaries}

\subsection{Basics of hyperkähler manifolds}

Given a $4 n$-dimensional real vector space $W$, we recall that a hypercomplex structure on $W$ is a triple $\left(I_{1}, I_{2}, I_{3}\right)$ of endomorphisms satisfying the multiplicative relations of the imaginary quaternions, $I_{\alpha}^{2}=-\operatorname{Id}_{W}$ and $I_{\alpha} I_{\beta}=I_{\gamma}$ for all cyclic permutations $(\alpha, \beta, \gamma)$ of $(1,2,3)$. Similarly, a hypercomplex structure on a $4 n$-dimensional real manifold $M$ is a triple $\left(J_{1}, J_{2}, J_{3}\right)$ of integrable complex structures on $M$, with the property that each triple $\left(I_{\alpha}:=\left.J_{\alpha}\right|_{x}\right), x \in M$, is a hypercomplex structure on $T_{x} M$.

These two notions are generalised as follows. Consider the three-dimensional subspace $Q_{W}$ of End $(W)$, which is the span $Q_{W}=\operatorname{span}_{\mathbb{R}}\left(I_{1}, I_{2}, I_{3}\right)$ of the endomorphisms of a hypercomplex structure $\left(I_{\alpha}\right)$. A (pseudo-)quaternionic Kähler structure on $W$ is an inner product $\langle\cdot, \cdot\rangle$ on $W$ which is Hermitian with respect to $Q_{W}$, that is, every element $J \in Q_{W}$ is skew-symmetric with respect to $\langle\cdot, \cdot\rangle$. For what concerns manifolds, we have instead the following

Definition 2.1 A 4n-dimensional (pseudo-)Riemannian manifold $(M, g)$ of signature $(4 p, 4 q), p+q=n$, is a (pseudo-)quaternionic Kähler ( $q k$ ) manifold if it admits a subbundle $Q \subset$ End $(T M)$ of quaternionic structures on the tangent spaces satisfying the following two conditions:

(i) each inner product $g_{x}$ is Hermitian for the quaternionic structure $Q_{x}$;

(ii) the parallel transport of the Levi-Civita connection $\nabla$ of $g$ preserves $Q$.

Further, $(M, g)$ is a (pseudo-)hyperkähler $(h k)$ manifold if there exist three global $\nabla$-parallel sections $J_{1}, J_{2}, J_{3}$ of $Q$ (which are thus integrable complex structures) determining a hypercomplex structure on each tangent space of $M$.

It is well known that a qk manifold is Einstein. Moreover, it is hk if and only if its scalar curvature is zero. In this paper we focus on hk manifolds, denoting them by a 5-tuple $\left(M, g, J_{\alpha}, \alpha=1,2,3\right)$, where the $J_{\alpha}$ are the $\nabla$-parallel sections generating the bundle $Q$.

An adapted frame at the point $x \in M$ of an hk manifold $\left(M, g, J_{\alpha}\right)$ is a $g$-orthonormal frame $u=\left(e_{A}\right): \mathbb{R}^{4 n} \rightarrow T_{x} M$ mapping the standard triple of complex structures $(\mathbf{i}, \mathbf{j}, \mathbf{k})$ of $\mathbb{H}^{n} \simeq \mathbb{R}^{4 n}$ into the triple of complex structures $\left(\left.J_{\alpha}\right|_{x}\right)_{\alpha=1,2,3}$. Note that any adapted frame $u=\left(e_{A}\right)$ allows the identification of the standard $\mathrm{Sp}_{1}$ - and $\mathrm{Sp}_{p, q}$-actions on $\mathbb{H}^{n}$ with uniquely associated actions of $\mathrm{Sp}_{1}$ - and $\mathrm{Sp}_{p, q}$-actions on $T_{x} M$. The family $O_{g}\left(M, J_{\alpha}\right)$ of all 
adapted frames is a principle bundle over $M$ with structure group $\operatorname{Sp}_{1} \cdot \mathrm{Sp}_{p, q}$ and is invariant under the parallel transport of the Levi-Civita connection. Hence, if the bundle $O_{g}\left(M, J_{\alpha}\right)$ admits a global section, the above actions of $\mathrm{Sp}_{1} \cdot \mathrm{Sp}_{p, q}$ on the tangent spaces $T_{x} M$ combine to yield a global action of $\mathrm{Sp}_{1} \cdot \mathrm{Sp}_{p, q}$ onto $T M$.

We now fix a few technical details, which we shall need.

As the standard representation of $\mathrm{Sp}_{1} \cdot \mathrm{Sp}_{p, q}$ on $\mathbb{H}^{n}=\mathbb{C}^{2 n}$, we choose the one for which the element $\left(\begin{array}{cc}i & 0 \\ 0 & -i\end{array}\right) \in \mathfrak{s p}_{1}$ acts on $\left(\mathbb{H}^{n}\right)^{\mathbb{C}} \simeq \mathbb{C}^{2} \otimes \mathbb{C}^{2 n}$ as the left multiplication by the complex structure $i$. Hence, for any given adapted frame $\left.u \in O_{g}\left(M, J_{\alpha}\right)\right|_{x}$, the corresponding action of $\left(\begin{array}{cc}i & 0 \\ 0 & -i\end{array}\right)$ on $T_{x} M$ coincides with the action of the complex structure $I=u_{*}(\mathbf{i})$. This action of $\mathrm{Sp}_{1} \cdot \mathrm{Sp}_{p, q}$ extends by $\mathbb{C}$-linearity to the standard action of $\mathrm{SL}_{2}(\mathbb{C}) \cdot \mathrm{Sp}_{n}(\mathbb{C})$ on $\left(\mathbb{H}^{n}\right)^{\mathbb{C}} \simeq \mathbb{C}^{2} \otimes \mathbb{C}^{2 n}$. Consequently, the $\mathbb{C}$-linear extensions $u:\left(\mathbb{H}^{n}\right)^{\mathbb{C}} \rightarrow T_{x}^{\mathbb{C}} M$ of adapted frames give $\mathrm{SL}_{2}(\mathbb{C}) \cdot \mathrm{Sp}_{n}(\mathbb{C})$-equivariant isomorphisms $T_{x}^{\mathbb{C}} M \simeq \mathbf{H}_{x} \otimes \mathbf{E}_{x}$ for complex vector spaces $\mathbf{H}_{x} \simeq \mathbb{C}^{2}$ and $\mathbf{E}_{x} \simeq \mathbb{C}^{2 n}$. These isomorphisms are necessarily related to each other by the action of some element of $\mathrm{Sp}_{1} \cdot \mathrm{Sp}_{p, q} \subset \mathrm{SL}_{2}(\mathbb{C}) \cdot \mathrm{Sp}_{n}(\mathbb{C})$.

Furthermore, as standard bases for $\mathbb{C}^{2}$ and $\mathbb{C}^{2 n}$, we shall use

$$
\left(h_{1}^{o}=(1,0), h_{2}^{o}=(0,1)\right), \quad\left(e_{a}^{o}=(0, \ldots, \underset{a \text {-th entry }}{1}, \ldots, 0)\right) .
$$

For any ( $\mathbb{C}$-linearly extended) adapted frame $u:\left(\mathbb{H}^{n}\right)^{\mathbb{C}}=\mathbb{C}^{2} \otimes \mathbb{C}^{2 n} \rightarrow T_{x}^{\mathbb{C}} M$, we then denote by $e_{i a}:=u\left(h_{i}^{o} \otimes e_{a}^{o}\right), i=1,2$, the complex vectors in $T_{x}^{\mathbb{C}} M$ corresponding to the basis $h_{i}^{o} \otimes e_{a}^{o}$ of $\mathbb{C}^{2} \otimes \mathbb{C}^{2 n}$. If $u: \mathbb{H}^{n} \rightarrow T_{x} M$ is changed into another adapted frame

$$
u^{\prime}=u \circ U \quad \text { with } \quad U=\left(\begin{array}{ll}
u_{+}^{1} & u_{-}^{1} \\
u_{+}^{2} & u_{-}^{2}
\end{array}\right) \in \operatorname{Sp}_{1} \subset \mathrm{SL}_{2}(\mathbb{C})=\operatorname{Sp}_{1}(\mathbb{C}),
$$

the corresponding complex basis $\left(e_{j a}\right)$ of $T_{x}^{\mathbb{C}} M$ is changed into

$$
e_{+a}=u_{+}^{j} e_{j a}, \quad e_{-a}=u_{-}^{i} e_{i a} .
$$

Similarly, if $u^{\prime}=u \circ U$ is further transformed by some $A=\left(A_{a}^{b}\right) \in \operatorname{Sp}_{p, q} \subset \operatorname{Sp}_{n}(\mathbb{C})$, the basis $\left(e_{ \pm a}\right)$ is then transformed into $e_{ \pm a}^{\prime}=A_{a}^{b} e_{ \pm b}$.

Note that, since the vectors $h_{1} \otimes e_{a}^{o}$ and $h_{2} \otimes e_{a}^{o}$ are $\pm i$-eigenvectors of the element $\left(\begin{array}{cc}i & 0 \\ 0 & -i\end{array}\right) \simeq \mathbf{i} \in \mathrm{Sp}_{1}$, the corresponding elements $e_{+a}, e_{-a} \in T_{x}^{\mathbb{C}} M$ are vectors of type $(1,0)$ and $(0,1)$ with respect to the complex structure $I=u(\mathbf{i})$ of $T_{x} M$.

\subsection{Connections, gauges, potentials and Yang-Mills fields}

In this section we briefly review certain basic facts about gauge theories which we shall need in what follows.

Given a manifold $M$ and a (principal or vector) bundle $\pi: E \rightarrow M$, we denote by $\mathfrak{X}(M)$ the space of all vector fields of $M$ and by $\Gamma(E)$ the set of all sections $\sigma: M \rightarrow E$. Given $X \in \mathfrak{X}(M)$ and a smooth (real or complex) function $f$, we write $X \cdot f$ to denote the directional derivative of $f$ along $X$.

Let $p: P \rightarrow M$ be a principal $G$-bundle and $p^{E}: E=P \times_{G, \rho} V \rightarrow M$ an associated vector bundle with fibre $V \simeq \mathbb{R}^{N}$, determined by a faithful linear representation $\rho: G \rightarrow \operatorname{GL}(V)$. We shall refer to an open subset $\mathcal{U} \subset M$ on which there exists a choice of gauge (local trivialisation) $\varphi:\left.P\right|_{\mathfrak{U}} \rightarrow \mathcal{U} \times G$ of the bundle $P$ over $\mathcal{U}$ as the domain of the gauge $\varphi$ (a minor abuse of the language). Given two gauges $\varphi, \varphi^{\prime}$ with overlapping domains $\mathcal{U}, \mathcal{U}^{\prime}$, the transition function $\varphi^{\prime} \circ \varphi^{-1}:\left(\mathcal{U} \cap \mathcal{U}^{\prime}\right) \times G \rightarrow\left(\mathcal{U} \cap \mathcal{U}^{\prime}\right) \times G$ is a map of the form 


$$
\varphi^{\prime} \circ \varphi^{-1}(x, h)=\left(x, g_{x} \cdot h\right) \text { for a smooth map } g: \mathcal{U} \rightarrow G .
$$

The family of the automorphisms of $G$ defined by $h \mapsto g_{x} \cdot h$ is what is usually called gauge transformation between the gauges $\varphi$ and $\varphi^{\prime}$. Finally, we recall that if a principal $G$-bundle $p: P \rightarrow M$ admits a collection of gauges, whose domains form an open cover of $M$ and whose associated gauge transformations $h \mapsto g_{x} \cdot h$ are determined by maps $g: \mathcal{U} \rightarrow G$ taking values in a fixed subgroup $G^{o} \subset G$, then such gauges determine a $G^{o}$-subbundle $p^{o}: P^{o} \rightarrow M$ of $P$, called $G^{o}$-reduction.

A connection 1-form $\omega$ on $P$ induces a unique covariant derivative on the associated bundle $E$. We recall that a covariant derivative on $E$ is an operator $D: \mathfrak{X}(M) \times \Gamma(E) \rightarrow \Gamma(E)$, which is $\mathbb{R}$-linear in both arguments, $\mathcal{C}^{\infty}(M ; \mathbb{R})$-linear in $\mathfrak{X}(M)$ and satisfies the Leibniz rule $D_{X}(f \sigma)=(X \cdot f) \sigma+f D_{X} \sigma$ for each $f \in \mathcal{C}^{\infty}(M ; \mathbb{R})$.

Now, consider a gauge $P \mid u \stackrel{\sim}{\rightarrow} \mathcal{U} \times G$ with domain $\mathcal{U}$. In this gauge, any vector in $T P \mid \mathcal{U}$ is naturally identified with a sum $X_{(x, g)}+B_{(x, g)} \in T_{(x, g)}(\mathcal{U} \times G) \simeq T_{x} \mathcal{U}+T_{g} G$ and the 1 -form $\omega$ on $\left.P\right|_{\mathcal{U}} \simeq \mathcal{U} \times G$ can be pointwise expressed as a sum of the form

$$
\left.\omega\right|_{(x, g)}=-A_{(x, g)}+\omega_{g}^{G},
$$

where $\omega^{G}$ is the Maurer-Cartan form of $G$ and, for each $g \in G$, the map $A_{(\cdot, g)}: \mathcal{U} \rightarrow$ $T^{*} \mathfrak{U} \otimes \mathfrak{g}$ is a $\mathfrak{g}$-valued 1 -form, which changes $G$-equivariantly with respect to $g$. The 1 -form $A:=A_{(\cdot, e)}: \mathcal{U} \rightarrow T^{*} M \otimes \mathfrak{g}$ is called the potential of $\omega$ in the considered gauge. If two gauges $\left.P\right|_{\mathfrak{U}} \simeq \mathcal{U} \times G,\left.P\right|_{\mathfrak{W}^{\prime}} \simeq \mathcal{U}^{\prime} \times G$ have overlapping domains $\mathcal{U}, \mathcal{U}^{\prime}$, the corresponding potentials $A, A^{\prime}$ are related through the gauge transformation $h \mapsto g_{x} \cdot h$ by means of

$$
A^{\prime}=\operatorname{Ad}_{g^{-1}} A+g^{-1} \cdot d g .
$$

We now recall that a section $\sigma: \mathcal{U} \rightarrow E \mid \mathcal{U} \simeq \mathcal{U} \times V$ of the associated bundle has the form $\sigma(x)=\left(x, s^{i}(x)\right)$ for a smooth map $\left(s^{i}\right): \mathcal{U} \rightarrow V=\mathbb{R}^{N}$. Hence, for each vector field $X \in \mathfrak{X}(\mathcal{U})$, it is possible to consider the section of $\left.E\right|_{\mathfrak{U}} \simeq \mathcal{U} \times V$

$$
D_{X} \sigma(x)=\left(x,\left.\left(X \cdot \sigma^{i}\right)\right|_{x}+\left.A_{j}^{i}(X) \sigma^{j}\right|_{x}\right),
$$

where $A_{j}^{i}:=\rho_{*} \circ A$ with $\rho_{*}: \mathfrak{g} \rightarrow \mathfrak{g l}(V)$ the Lie algebra representation determined by the linear representation $\rho: G \rightarrow \operatorname{GL}(V)$ that gives the associated vector bundle $E=P \times_{G, \rho} V$. The $\mathfrak{g l}(V)$-valued 1 -form $A_{j}^{i}$ is called the potential of $D$ induced by the connection $\omega$. The operator (2.3) is gauge independent due to (2.2), thus globally well defined as a covariant derivative on the sections of $E$.

We recall that the curvature 2-form of $\omega$ is the $\mathfrak{g}$-valued 2-form on $P$ defined by $\Omega=$ $d \omega+\frac{1}{2}[\omega, \omega]$. Given a gauge $\varphi: P \mid \mathfrak{u} \rightarrow \mathcal{U} \times \mathfrak{g}$ and the associated gauge $\widehat{\varphi}$ for $E$

$$
\left.\widehat{\varphi}:\left.E\right|_{u} \rightarrow \mathcal{U} \times V, \quad \widehat{\varphi}\left(\left[\varphi^{-1}(x, e), v\right)\right]\right):=(x, v),
$$

the curvature tensors of $\omega$ and of $D$ are the ( $\mathfrak{g}$ - or $\mathfrak{g l}(V)$-valued) 2 -forms $\mathcal{F}^{\varphi}$ and $F_{x}$ on $\mathcal{U}$, defined by

$$
\mathcal{F}_{x}^{\varphi}(v, w):=2 \Omega_{(x, e)}(v, w), \quad F_{x}^{\widehat{\varphi}}(v, w):=2 \rho_{*} \circ \mathcal{F}_{x}^{\varphi}(v, w), \quad \text { for } \quad v, w \in T_{x} \mathcal{U} .
$$

Note that $\mathcal{F}^{\varphi}$ can be recovered from the potential $A$ of $\omega$ by the formula

$$
\mathcal{F}^{\varphi}(X, Y)=X \cdot(A(Y))-Y \cdot(A(X))+[A(X), A(Y)]-A([X, Y]) .
$$

and that, if $h \mapsto g_{x} \cdot h$ is the gauge transformation between $\varphi, \varphi^{\prime}$, one has that

$$
\mathcal{F}_{x}^{\varphi^{\prime}}=\operatorname{Ad}_{g_{x}} \mathcal{F}_{x}^{\varphi}, \quad F_{x}^{\widehat{\varphi}^{\prime}}=F_{x}^{\widehat{\varphi}} .
$$


This shows that the curvature tensor of $\omega$ does depend on the gauge, while the curvature tensor of $D$ does not. Due to this, the curvatures $F_{x}^{\widehat{\varphi}}$ combine and determine a globally defined $\mathfrak{g l}(V)$-valued 2-form $F$ on $M$. It can be also checked that it satisfies the identity

$$
F(X, Y) s=\left[D_{X}, D_{Y}\right] s-D_{[X, Y]} s \text { for sections } s \in \Gamma(E),
$$

a property often used as an alternative definition of the curvature of $D$.

All of the above notions and properties have analogues in the case of holomorphic bundles, which we now briefly recall.

If $(M, J)$ is a complex manifold, $G$ is a complex Lie group and $V$ is a complex vector space, a principal $G$-bundle $p: P \rightarrow M$ (resp. a complex vector bundle $p: E \rightarrow M$ with fibre $V$ ) is called holomorphic if it is equipped with a complex structure $\widehat{J}$, such that the right action of $G$ on $P$ (resp. the vector bundle structure on $E$ ) is $\widehat{J}$-holomorphic and the projection $p$ is a $(\widehat{J}, J)$-holomorphic mapping. In this case, a trivialisation is called holomorphic if it is a local holomorphic map from $(P, \widehat{J})(\operatorname{resp} .(E, \widehat{J}))$ to the Cartesian product $M \times G$ (resp. $M \times V)$, equipped with the product complex structure.

A connection form $\omega$ on a holomorphic $G$-bundle $(P, \widehat{J})$ is called $\widehat{J}$-invariant if the corresponding horizontal spaces $\mathcal{H}_{(x, g)}=\operatorname{ker} \omega_{(x, g)}$ are invariant under the complex structure $\widehat{J}$. This is equivalent to say that, in any holomorphic trivialisation $\varphi:\left.P\right|_{\mathcal{U}} \rightarrow \mathcal{U} \times G$, the corresponding potential $A: \mathcal{U} \rightarrow T^{*} \mathcal{U} \otimes \mathfrak{g}$ takes actually values in $T^{10 *} M \otimes \mathfrak{g}^{10}+T^{01 *} M \otimes \mathfrak{g}^{01}$. Here we denote by $T_{x}^{10} M$ and $T^{01} M$ the holomorphic and anti-holomorphic distributions of $M$ and by $\mathfrak{g}^{10}, \mathfrak{g}^{01}$ the subalgebras of $\mathfrak{g}^{\mathbb{C}}$ (both isomorphic to $\mathfrak{g}$ ), which are generated by the vectors of type $(1,0)$ and $(0,1)$, respectively. Being each $A_{x}, x \in \mathcal{U}$, real, the projection of $A_{x}$ onto $T_{x}^{01 *} M \otimes \mathfrak{g}^{01}$ is the complex conjugate of the component in $T_{x}^{10 *} M \otimes \mathfrak{g}^{10}$. So, the potential $A$ is uniquely determined by the associated map $A^{10}: \mathcal{U} \rightarrow T^{10 *} M \otimes \mathfrak{g}^{10}$, which we call $(1,0)$-potential.

We finally remark that the covariant derivative $D$ on an associated holomorphic vector bundle $p^{E}: E \rightarrow M$, determined by a $\widehat{J}$-invariant connection $\omega$, is characterised by the property that it transforms sections of $E^{\mathbb{C}}=E^{10} \oplus_{M} E^{01}$, with values in $E^{10}$ or in $E^{01}$, into sections which are still in $E^{10}$ or in $E^{01}$, respectively. The covariant derivatives of $(1,0)$ type (or $(0,1)$-type) sections and the change of $(1,0)$-potentials under holomorphic gauge transformations behave exactly as in formulas (2.3) and (2.2).

We conclude this short section by recalling the definition of gauge fields. A gauge field with structure group $G$ is a pair $(E, D)$, formed by:

(1) a vector bundle $E$, associated with a principal $G$-bundle $p: P \rightarrow M$,

(2) a covariant derivative $D$ on $E$, induced by a connection $\omega$ on $P$.

In this case we say that $(E, D)$ is a gauge field associated with the pair $(P, \omega)$. If $G$ is complex, $p: P \rightarrow M$ admits a reduction to a $G^{o}$ - subbundle $P^{o} \subset P$ with $G^{o}$ compact real form of $G$ and $\omega$ restricts to a connection $\omega^{o}$ on $P^{o}$, we say that $(P, \omega)$ is reducible to $\left(P^{o}, \omega^{o}\right)$ and that $(E, D)$ is the complexification of a gauge field with compact structure group $G^{o}$.

If $M$ is an oriented (pseudo-)Riemannian manifold, we may define the Hodge *-operator, $*: \Lambda^{p} T^{*} M \rightarrow \Lambda^{n-p} T^{*} M$. Then, the gauge field $(E, D)$ is called a Yang-Mills field if its curvature tensor $F$ satisfies the Yang-Mills equation $D * F=0$.

\section{$2.3 \mathcal{C}^{k}$-norms and $L^{p}$-norms of curvatures}

Let $G$ be a reductive complex Lie group and $G^{o} \subset G$ a compact real form of $G$. Further let $\mathfrak{g}^{o}=\mathfrak{g}^{s}+\mathfrak{z}\left(\mathfrak{g}^{o}\right)$ be the decomposition of $\mathfrak{g}^{o}=\operatorname{Lie}\left(G^{o}\right)$ into its semisimple part $\mathfrak{g}^{S}$ and 
centre $\mathfrak{z}\left(\mathfrak{g}^{o}\right)$, and denote by $\langle\cdot, \cdot\rangle$ any $\operatorname{Ad}_{G_{o}}$-invariant Euclidean inner product on $\mathfrak{g}^{o}$ which, on $\mathfrak{g}^{s} \times \mathfrak{g}^{s}$, coincides with minus the Cartan-Killing form. This allows us to define the Hermitian inner product on $\mathfrak{g}=\operatorname{Lie}(G)=\mathfrak{g}^{o}+i \mathfrak{g}^{o}$

$$
h: \mathfrak{g} \times \mathfrak{g} \rightarrow \mathbb{C}, \quad h\left(X+i Y, X^{\prime}+i Y^{\prime}\right)=\left\langle X+i Y, X^{\prime}-i Y^{\prime}\right\rangle
$$

and the associated norm $\|X+i Y\|_{h}=\sqrt{\|X\|_{\langle\cdot, \cdot\rangle}^{2}+\|Y\|_{\langle,,\rangle}^{2}}$. If $(M, g)$ is a Riemannian manifold, we can use the metric $g$ to extend the Hermitian product $h$ of $\mathfrak{g}$ to a positive inner product, also denoted by $h$, on the space of tensor fields in $\otimes^{\ell} T^{*} M \otimes \mathfrak{g}$ over $M$. So, for any compact subset $K \subset M$, we have the usual sup-norm for $\mathfrak{g}$-valued $\mathcal{C}^{k}$ functions on $K$

$$
\|V\|_{\mathcal{C}^{k}(K, \mathfrak{g})}:=\sum_{j=0}^{k} \sup _{x \in K}\left\|\left.\nabla^{j} V\right|_{x}\right\|_{h} .
$$

Similarly, for each $p \in[1,+\infty)$, we may use the $L^{p}$-space $L^{p}(\mathcal{U}, \mathfrak{g})$, the completion of the space of all $\mathcal{C}^{0}$-maps $V: \mathcal{U} \rightarrow \mathfrak{g}$ with bounded values for the integral $\int_{\mathcal{U}}\|V\|_{h}^{p} \operatorname{vol}_{g}$, equipped with the usual $L^{p}$-norm

$$
\|\cdot\|_{L^{p}(U)}:=\left(\int_{U}\|\cdot\|_{h}^{p} \operatorname{vol}_{g}\right)^{\frac{1}{p}} .
$$

All such norms immediately generalise to spaces of $\mathfrak{g}$-valued $r$-forms.

Consider now a gauge field $(E, D)$ associated with the pair $(P, \omega)$ and a trivialisation $\varphi:\left.P\right|_{\mathcal{U}} \rightarrow \mathcal{U} \times G$ in which the curvature tensors of $\omega$ and $D$ are $\mathcal{F}^{\varphi}$ and $F=F^{\widehat{\varphi}}$, respectively. Then, for any compact subset $K \subset \mathcal{U}$, we define

$$
\|F\|_{\mathcal{C}^{k}(K)}^{(\varphi)}:=\left\|\mathcal{F}^{\varphi}\right\|_{\mathcal{C}^{k}(K, \mathfrak{g})}, \quad\|F\|_{L^{p}(\mathcal{U})}^{(\varphi)}:=\left\|\mathcal{F}^{\varphi}\right\|_{L^{p}(\mathcal{U}, \mathfrak{g})} .
$$

Now consider the cases when $(P, \omega)$ is reducible to a pair $\left(P^{o}, \omega^{o}\right)$ with structure group given by the compact $G^{o}$. Since the $h$-norms are $\operatorname{Ad}_{G_{o}}$-invariant, if we consider only the gauges which determine such a reduction, then the norms (2.9) do not depend on $\varphi$ and they coincide with the usual $\mathcal{C}^{k}$ - and Sobolev norms of curvatures for gauge fields with compact structure groups.

\subsection{Lifting gauge fields}

Let $(E, D)$ be a gauge field on $M$ associated with a pair $(P, \omega)$. If $\pi: N \rightarrow M$ is a principal $H$-bundle over $M$, the lift of $(P, \omega)$ is the pair $\left(P^{\prime}, \omega^{\prime}\right)$ given by

(a) the lifted $G$-bundle $p^{\prime}: P^{\prime}:=\pi^{*} P \rightarrow N$, i.e. the submanifold

$$
\pi^{*} P:=\{(y, U) \in N \times P \text { such that } p(U)=\pi(y)\} \subset N \times P
$$

equipped with the natural projection $p^{\prime}: \pi^{*} P \rightarrow N, p^{\prime}(y, u):=y$

(b) the pull-back connection $\omega^{\prime}:=\pi^{\prime *} \omega$ on $P^{\prime}=\pi^{*} P$ determined by the projection $\pi^{\prime}: P^{\prime} \subset N \times P \rightarrow P$.

The lift of the gauge field $(E, D)$ is the gauge field $\left(E^{\prime}=\pi^{*} E, D^{\prime}=\pi^{*} D\right)$ on $N$ given by

(i) the lifted vector bundle $q^{\prime}: E^{\prime}=\pi^{*} E=P^{\prime} \times_{G, \rho} V \rightarrow N$ over $N$

(ii) the covariant derivative $D^{\prime}=\pi^{*} D$ on $E^{\prime}$ induced by $\omega^{\prime}$. 
We now briefly discuss the problem of characterising the gauge fields $\left(E^{\prime}, D^{\prime}\right)$ on principal $H$-bundles $\pi: N \rightarrow M$ which are lifts of gauge fields on $M$. Given $\pi: N \rightarrow M$ and $(E, D)$ as above, for each $X \in \mathfrak{h}=\operatorname{Lie}(H)$, the corresponding 1-parameter subgroup $\exp (\mathbb{R} X) \subset H$ has clearly a natural right action on $N$ and determines a natural 1-parameter group of diffeomorphisms on the lifted bundle $P^{\prime} \subset N \times P$ given by

$$
R^{\prime}: \mathbb{R} \longrightarrow \operatorname{Diff}\left(P^{\prime}\right), \quad R_{t}^{\prime}(y, u)=\left(R_{\exp (t)}(y), u\right) .
$$

Note that each map $R_{t}^{\prime}$ is a bundle automorphism that preserves the connection $\omega^{\prime}$ and induces a bundle automorphism of the associated vector bundle $E^{\prime}=\pi^{*} E$ that commutes with the covariant derivative $D^{\prime}=\pi^{*} D$. Therefore, the vector field $X^{\prime}$ on $P^{\prime}$, whose flow is the 1-parameter group of automorphisms $R_{t}$, is such that

(a) $p_{*}^{\prime}\left(X^{\prime}\right)=X$ and $\pi_{*}^{\prime}\left(X^{\prime}\right)=0$; here $X$ is identified with the vertical vector field of the $H$-bundle $N$

(b) $\imath_{X^{\prime}} \omega^{\prime}=0$ and $0=\mathcal{L}_{X^{\prime}} \omega^{\prime}=\imath_{X^{\prime}} d \omega^{\prime}+d\left(\imath_{X^{\prime}} \omega^{\prime}\right)=\imath_{X^{\prime}} d \omega^{\prime}$;

(c) if $A$ is the potential of $\omega^{\prime}$ in a gauge $\pi^{*} P \mid u \simeq \mathcal{U} \times G$, the vector field $X^{\prime}$ has the form $X_{(y, g)}^{\prime}=X_{y}+\left.X^{\mathfrak{g}(y)}\right|_{g}$ where $X^{\mathfrak{g}(y)}$ is the left invariant vector field of $G$ defined by $\omega^{G}\left(X^{\mathfrak{g}(y)}\right)=A\left(X_{y}\right)$.

Note that (b) implies also that

$$
\imath_{X^{\prime}} \Omega^{\prime}=0 \text { and hence that } \imath_{X} F^{\prime}=0 \text { for } X \in \mathfrak{k} .
$$

All this has the following converse.

Proposition 2.2 Let $\left(E^{\prime}, D^{\prime}\right)$ be a gauge field associated with a pair $\left(P^{\prime}, \omega^{\prime}\right)$ on an $H$-bundle $\pi: N \rightarrow M$ and, for any given gauge with domain $U \subset N$, denote by $\mathcal{F}^{\prime}, F^{\prime}$ the curvature tensors of $\omega^{\prime}$ and $D^{\prime}$.

If $H$ is simply connected, then $\left(E^{\prime}, D^{\prime}\right)$ is the lift of a gauge field on $M$ if and only if, for each $X \in \mathfrak{h}$, the associated infinitesimal transformation on $N$ is such that a) $\iota_{X} \mathcal{F}^{\prime}=0$ or, equivalently, $\iota_{X} F^{\prime}=0$ in any gauge and $b$ ) the unique $\omega^{\prime}$-horizontal vector field $X^{\prime}$ on $P^{\prime}$, which projects onto $X$, is complete.

Proof The necessity follows from previous remarks. Assume now that for each $X \in \mathfrak{h}$, conditions (a) and (b) hold. Since $l_{X^{\prime}} \omega^{\prime}=0$, we have that $\mathcal{L}_{X^{\prime}} \omega^{\prime}=l_{X^{\prime}} d \omega^{\prime}+d\left(l_{X^{\prime}} \omega^{\prime}\right)=$ $\imath_{X^{\prime}} \Omega^{\prime}=0$, from which it follows that the flow of $X^{\prime}$ commutes with the right $G$-action of $P^{\prime}$ and preserves $\omega^{\prime}$. We also have that, for any $X, Y \in \mathfrak{h}$, their $\omega^{\prime}$-horizontal lifts $X^{\prime}, Y^{\prime}$ are such that $\omega^{\prime}\left(\left[X^{\prime}, Y^{\prime}\right]\right)=\Omega^{\prime}\left(X^{\prime}, Y^{\prime}\right)=0$. This means that $\left[X^{\prime}, Y^{\prime}\right]$ is the $\omega^{\prime}$-horizontal lift of the Lie bracket $[X, Y]$. We therefore conclude that the collection of vector fields

$$
\mathfrak{h}^{\prime}:=\left\{X^{\prime} \text { is } \omega^{\prime} \text {-horizontal lift of some } X \in \mathfrak{h}\right\}
$$

is a finite-dimensional Lie algebra of complete vector fields on $P^{\prime}$. By a classical theorem of Palais [32], this implies the existence of a right $H$-action on $P^{\prime}$ whose infinitesimal transformations are precisely the $\omega^{\prime}$-horizontal vector fields $X^{\prime} \in \mathfrak{h}^{\prime}$. All orbits of this action are regular and simply connected, because all of them are coverings of the simply connected $H$-orbits on $N$. Moreover, each transformation of this action is a bundle automorphism, which preserves the connection 1-form $\omega^{\prime}$ and commutes with the $G$-action. Hence, the orbit space $P=P^{\prime} / H$ is a $G$-bundle over $M:=P^{\prime} / H^{\prime} \times G=P / G$ and is equipped with the $\mathfrak{g}$-valued 1-form $\omega$ defined by

$$
\omega_{[u]}(v):=\omega_{u}^{\prime}\left(v^{\prime}\right) \text { for some } v^{\prime} \in T_{u} P^{\prime} \text { that projects onto } v \in T_{[u]} P^{\prime} / H^{\prime} .
$$


One can directly check that $\omega$ is a connection and that $\omega^{\prime}$ is the pull-back of $\omega$ on $P^{\prime}$. The associated bundle $E$ of $P \rightarrow M=P / G$, equipped with the covariant derivation $D$ determined by $\omega$, is the desired gauge field, of which $\left(E^{\prime}, D^{\prime}\right)$ is a lift.

\section{Harmonic spaces of (pseudo-)hyperkähler manifolds}

In the rest of this paper, $\left(M, g, J_{\alpha}\right)$ denotes an hk manifold and $(E, D)$ a complex gauge field on $\left(M, g, J_{\alpha}\right)$ associated with a pair $(P, \omega)$ with complex structure group $G$. We shall mostly assume that $G$ is the complexification of a compact real form $G^{o}$ and that $(P, \omega)$ is the complexification of a pair $\left(P^{o}, \omega^{o}\right)$, with structure group $G^{o}$. We shall simply say that $(E, D)$ is the "complexification" of a gauge field having compact structure group $G^{o}$.

\subsection{The twistor bundle of a (pseudo-)hyperkähler manifold}

Let $\left(M, g, J_{\alpha}\right)$ be a $4 n$-dimensional hk manifold. It is well known that for a point $z=$ $(a, b, c) \in S^{2}\left(\simeq \mathbb{C} P^{1}\right)$, the tensor field $I^{(z)}:=a J_{1}+b J_{2}+c J_{3}$ is an integrable complex structure on $M$ and that the twistor bundle $Z(M)$ of $M$ is simply the trivial $\mathbb{C} P^{1}$-bundle $Z(M):=M \times\left\{I^{(z)}, z \in S^{2}\right\} \rightarrow M$. Since each $z \in S^{2}$ corresponds to a distinct integrable complex structure, the twistor bundle $Z(M)$ is foliated by the complex submanifolds $M \times$ $\{z\} \simeq M$, each equipped with the corresponding complex structure $I^{(z)}$. Such complex structures combine with the classical complex structure of $\mathbb{C} P^{1}$ and determine a natural almost complex structure on $Z(M)$, which we denote by $\widehat{I}$. It was proved to be integrable in [34].

We remark that the complex structures on $T_{x} M, x \in M$,

$$
\left.I^{(z)}\right|_{x}:=\left.a J_{1}\right|_{x}+\left.b J_{2}\right|_{x}+\left.c J_{3}\right|_{x}, \quad z=(a, b, c) \in S^{2} \simeq \mathbb{C} P^{1},
$$

coincide with the complex structures of the form $I=u_{*}(\mathbf{i}) \in \operatorname{span}\left(I_{\alpha}:=\left.J_{\alpha}\right|_{x}\right)$, given by adapted frames $u$ of $T_{x} M$ as mentioned in Sect. 2.1. It follows that each of the $2 n$-tuples of complex vectors $e_{+a}$ (resp. $e_{-a}$ ), which are part of the complex bases (2.1), is actually a frame of holomorphic (resp. anti-holomorphic) vectors for a complex leaf $M \times\left\{I^{(z)}\right\} \subset Z(M)$.

\subsection{The harmonic space of a (pseudo-)hyperkähler manifold}

The harmonic space of a $4 n$-dimensional hk manifold $\left(M, g, J_{\alpha}\right)$ is the trivial bundle $\mathcal{H}(M)=M \times \mathrm{SL}_{2}(\mathbb{C}) \rightarrow M$, endowed with an integrable complex structure $\mathbb{I}$ defined as follows. For each point $(x, U) \in \mathcal{H}(M)$, consider the natural direct sum decomposition $T_{(x, U)} \mathcal{H}(M)=T_{x} M+T_{U} \mathrm{SL}_{2}(\mathbb{C}) \simeq T_{x} M+\mathfrak{s l}_{2}(\mathbb{C})$, where $T_{U} \mathrm{SL}_{2}(\mathbb{C})$ is identified with $\mathfrak{s l}_{2}(\mathbb{C})$ by means of right invariant vector fields. Then let $\mathbb{I}_{(x, U)}$ be the unique complex structure on $T_{(x, U)} \mathcal{H}(M)$ given by

$$
\left.\mathbb{I}_{(x, U)}\right|_{T_{x} M}:=\left.I^{(z)}\right|_{x}, z=U \cdot[0: 1] \in \mathbb{C} P^{1}\left(\simeq S^{2}\right) \text { and }\left.\mathbb{I}_{(x, U)}\right|_{\mathfrak{s} l_{2}(\mathbb{C})}=J_{o},
$$

where $J_{o}$ is the complex structure of $\mathfrak{s l}_{2}(\mathbb{C})$ given by the multiplication by $\left(\begin{array}{ll}i & 0 \\ 0 & i\end{array}\right)$. From the above identification $T_{U} \mathrm{SL}_{2}(\mathbb{C}) \simeq \mathfrak{s l}_{2}(\mathbb{C})$, it follows that along each fibre $\{x\} \times \mathrm{SL}_{2}(\mathbb{C})$, the $\mathbb{I}$-holomorphic distribution is generated by right invariant vector fields of $\mathrm{SL}_{2}(\mathbb{C})$.

The collection $\mathbb{I}$ of such pointwise defined complex structures is a globally defined almost complex structure on $\mathcal{H}(M)$, which can be seen to be integrable as follows. The family of 
restricted complex structures $\left.\mathbb{I}\right|_{M \times\{U\}}=\left.I^{(z)}\right|_{M \times\{U\}}$ on the manifolds $M \times\{U\}, U \in \mathrm{SL}_{2}(\mathbb{C})$, is invariant under the natural left action of $\mathrm{SL}_{2}(\mathbb{C})$ on $\mathcal{H}(M)$. Thus, the Lie derivative of an $\mathbb{I}$-holomorphic vector field that is tangent to the (horizontal) slices $M \times\{U\}$ by means of an infinitesimal transformations of this $\mathrm{SL}_{2}(\mathbb{C})$-action always gives another $\mathbb{I}$-holomorphic vector field, which is horizontal. On the other hand, the infinitesimal transformations of the left action of $\mathrm{SL}_{2}(\mathbb{C})$ on each vertical fibre $\{x\} \times \mathrm{SL}_{2}(\mathbb{C}) \simeq \mathrm{SL}_{2}(\mathbb{C})$ are nothing but the right invariant vector fields of $\mathrm{SL}_{2}(\mathbb{C})$. This implies that the Lie bracket between a horizontal $\mathbb{I}$-holomorphic vector field and a vertical $\mathbb{I}$-holomorphic vector field is a horizontal I-holomorphic vector field. This property together with the fact that both the horizontal and vertical $\mathbb{I}$-holomorphic distributions are involutive proves that the whole $\mathbb{I}$-holomorphic distribution of $\mathcal{H}(M)$ is involutive, i.e. that $\mathbb{I}$ is globally integrable.

Remark 3.1 Note that $\mathcal{H}(M)$, considered as a principal $\mathrm{SL}_{2}(\mathbb{C})$-bundle over $M$, can be (locally) identified with a bundle of vertical complex frames $\left(e_{+}, e_{-}\right)$for the fibres of the rank 2 holomorphic vector bundle $\pi: \mathbf{H} \rightarrow M$ introduced by Salamon [34] for general quaternionic Kähler manifolds. (Note that the fibres of $\mathbf{H}$ are the spaces $\mathbf{H}_{x}$ defined in Sect. 2.1). The explicit construction of the complex structures of $\mathcal{H}(M)$ and $\mathbf{H}$ directly yields that the projection

$$
p: \mathcal{H}(M) \rightarrow \mathbf{H} \backslash\{0\}, \quad p\left(\left(x,\left(e_{+}, e_{-}\right)\right)=e_{+} \in \mathbf{H}_{x}, \quad x \in M\right.
$$

is holomorphic. Thus, $\mathcal{H}(M)$ fibres holomorphically over the twistor space $Z(M)=P(\mathbf{H}) \simeq$ $M \times \mathbb{C} P^{1}$ with typical fibre given by the subgroup $B \subset \mathrm{SL}_{2}(\mathbb{C})$ of upper triangular matrices. Thus, $Z(M) \simeq \mathcal{H}(M) / B=M \times \mathrm{SL}_{2}(\mathbb{C}) / B$.

By construction, the harmonic space $\mathcal{H}(M)$ is equipped with the $\mathbb{I}$-invariant integrable distribution $\mathcal{D}$ given by the tangent spaces of the leaves $M \times\{U\}$. The two complex subdistributions of $\mathcal{D}^{\mathbb{C}}$, spanned by the holomorphic and anti-holomorphic vector fields, will be denoted by $\mathcal{D}_{+}, \mathcal{D}_{-} \subset \mathcal{D}^{\mathbb{C}}$. By the remarks at the end of Sect. 3.1, for $(x, U) \in \mathcal{H}(M)$ there is at least one adapted frame $u: \mathbb{H}^{n} \rightarrow T_{x} M$, more precisely, a frame with $u(\mathbf{i})=I_{x}^{(z)}$, $z=U \cdot[0: 1]$, such that the corresponding $2 n$-tuples $\left(e_{+a}\right)$ and $\left(e_{-a}\right)$ are bases for the vector spaces $\left.\mathcal{D}_{+}\right|_{(x, U)}$ and $\left.\mathcal{D}_{-}\right|_{(x, U)}$, respectively.

\subsection{The complexified harmonic space}

Consider an $n$-dimensional complex manifold $(N, J)$ and denote by $\mathcal{A}_{J}$ the complete atlas of holomorphic coordinates, i.e. of systems of coordinates $\xi=\left(z^{i}\right): \mathcal{U} \subset N \rightarrow \mathbb{C}^{n}$ in which the integrable complex structure $J$ has the standard form $J=i \frac{\partial}{\partial z^{j}} \otimes d z^{j}-i \frac{\partial}{\partial \bar{z}^{k}} \otimes d \bar{z}^{k}$. We define the complexification of $(N, J)$ as the pair $\left(N^{\mathbb{C}}, l\right)$ given by:

(a) the complex manifold $N^{\mathbb{C}}:=N \times N$ having complex structure $\widetilde{J}$ defined at each point $(x, y) \in N \times N$ by $\widetilde{J}_{(x, y)}(v, w):=J_{x}(v)-J_{y}(w)$

(b) the standard diagonal embedding $\imath: N \rightarrow N^{\mathbb{C}}, l(x)=(x, x)$.

Note that the complex structure $\widetilde{J}$ of $N^{\mathbb{C}}$ is defined in such a way that the corresponding atlas $\mathcal{A}_{\widetilde{J}}$ of holomorphic coordinates is generated by coordinates of the form $\widetilde{\xi}=\left(z^{i}, \overline{z^{\prime} j}\right)$ : $\mathcal{U} \times \mathcal{V} \rightarrow \mathbb{C}^{2 n}$ for some (local) holomorphic coordinates $\left(z^{i}\right),\left(z^{\prime}\right)$ of $(N, J)$. So, the antiholomorphic involution $\tau: N^{\mathbb{C}} \rightarrow N, \tau(x, y)=(y, x)$ has a fixed point set which is precisely the totally real submanifold $l(N) \simeq N$.

The above construction yields the following very convenient extension of harmonic spaces. Let $\mathcal{H}^{\mathbb{C}}(M)$ be the Cartesian product $\mathcal{H}^{\mathbb{C}}(M)=M \times M \times \mathrm{SL}_{2}(\mathbb{C})$, equipped with the unique 
(integrable) complex structure $\mathbb{I}^{(\mathbb{C})}$, which coincides with the right invariant complex structure along the (vertical) leaves $\{x\} \times\{y\} \times \mathrm{SL}_{2}(\mathbb{C}) \simeq \mathrm{SL}_{2}(\mathbb{C})$ (see (3.1)) and with the complex structure of the complexification of $\left(M, I^{(z)}\right), z=U \cdot[0: 1]$, along each horizontal leaf $M \times M \times\{U\}$. In other words, $\mathcal{H}^{\mathbb{C}}(M)$ is the union of the complexifications of the manifolds $\left(M, I^{(z)}\right), z \in S^{2}$, and not the complexification of the harmonic space $\mathcal{H}(M)$. Nevertheless, we call $\mathcal{H}^{\mathbb{C}}(M)$ the complexified harmonic space.

Both of the plus and minus distributions $\mathcal{D}_{ \pm} \subset T^{\mathbb{C}} \mathcal{H}(M)$ naturally extend to holomorphic distributions on $\mathcal{H}^{\mathbb{C}}(M)$. In order to see this, just consider the distribution $\mathcal{D}^{\prime}$ on $\mathcal{H}^{\mathbb{C}}(M)$, determined by the tangent spaces to all leaves $M \times M \times\{U\}$ and the associated complex subdistribution $\mathcal{D}_{\mathbb{I}^{(\mathbb{C})}}^{10} \subset \mathcal{D}^{\prime \mathbb{C}}$ spanned by $\mathbb{I}^{(\mathbb{C})}$-holomorphic vector fields. The subdistributions of $\mathcal{D}_{\mathbb{I}(\mathbb{C})}^{10}$ that project isomorphically onto the tangent spaces either on the first or the second copy of $M$ coincide with the distributions $\mathcal{D}_{ \pm}$at the points of the real submanifold $\mathcal{H}(M) \subset$ $\mathcal{H}^{\mathbb{C}}(M)$. For simplicity, we shall denote these subdistributions of $\mathcal{D}_{\mathbb{I}}^{10}$ al also by $\mathcal{D}_{ \pm}$.

\section{Instantons on hk manifolds and prepotentials}

\subsection{Instantons on hyperkähler manifolds}

As usual, let $\left(M, g, J_{\alpha}\right)$ be an hk manifold and denote by $T_{x}^{\mathbb{C}} M \simeq \mathbf{H}_{x} \otimes \mathbf{E}_{x}, x \in M$, the isomorphisms described in Sect. 2.1. The space of complex 2-forms $\Lambda^{2} T_{x}^{* \mathbb{C}} M$ splits into three irreducible $\mathrm{SL}_{2}(\mathbb{C}) \cdot \mathrm{Sp}_{n}(\mathbb{C})$ moduli:

$$
\Lambda^{2} T_{x}^{* \mathbb{C}} M \simeq \mathbb{C} \omega_{\mathbf{H}_{x}} \otimes S^{2} \mathbf{E}_{x}^{*}+S^{2} \mathbf{H}_{x}^{*} \otimes \mathbb{C} \omega_{\mathbf{E}_{x}}+S^{2} \mathbf{H}_{x}^{*} \otimes \Lambda_{0}^{2} \mathbf{E}_{x}^{*}
$$

Here $\omega_{\mathbf{H}_{x}}$ and $\omega_{\mathbf{E}_{x}}$ are the $\mathrm{SL}_{2}(\mathbb{C})$ - and $\mathrm{Sp}_{n}(\mathbb{C})$-invariant symplectic forms of $\mathbf{H}_{x}$ and $\mathbf{E}_{x}$, respectively, and $\Lambda_{0}^{2} \mathbf{E}_{x}$ is the irreducible $\mathrm{Sp}_{n}(\mathbb{C})$-submodule of $\Lambda^{2} \mathbf{E}_{x}$ complementary to $\mathbb{C} \omega_{\mathbf{E}_{x}}$. Since the isomorphism $T_{x}^{\mathbb{C}} M \simeq \mathbf{H}_{x} \otimes \mathbf{E}_{x}$ is unique up to an action of an element in $\mathrm{Sp}_{1} \cdot \mathrm{Sp}_{p, q}$, the decomposition (4.1) is independent of the isomorphism chosen.

Now, given a gauge field $(E, D)$ on an hk manifold, we split the (C-linear extension of the) curvature tensor $F_{x}, x \in M$ as follows:

$$
\begin{aligned}
F_{x}=F_{x}^{(1)}+F_{x}^{(2)} \text { with } \quad F_{x}^{(1)} \in \mathbb{C} \omega \mathbf{H}_{x} \otimes S^{2} \mathbf{E}_{x}^{*} \otimes \text { End }\left(E_{x}\right) \\
F_{x}^{(2)} \in\left(S^{2} \mathbf{H}_{x}^{*} \otimes\left(\mathbb{C} \omega_{\mathbf{E}_{x}}+\Lambda_{0}^{2} \mathbf{E}_{x}^{*}\right)\right) \otimes \text { End }\left(E_{x}\right) .
\end{aligned}
$$

A gauge field $(E, D)$ is called instanton if the $F^{(2)}$ component of its curvature tensor vanishes everywhere. Such instantons provide minima of the Yang-Mills functional $\int_{M}|F|^{2} \omega_{g}$ and are thus solutions of the Yang-Mills equations [41]. Such instanton equations have been studied by several authors $[1,9,12,28,34,36]$.

Notice that the vanishing of $F^{(2)}$ corresponds to simple conditions on the components $F_{ \pm a \mid \pm b}=F\left(e_{ \pm a}, e_{ \pm b}\right)$ with respect to the complex frames $\left(e_{ \pm a}\right)$ defined in (2.1). In fact, $F^{(2)}=0$ if and only if

$$
F_{+a \mid+b}=F_{-a \mid-b}=0, \quad F_{+a \mid-b}=-F_{-a \mid+b}, \quad F_{+a \mid-b}=F_{+b \mid-a} .
$$

In four dimensions these are precisely the well-known anti-self-duality equations. 


\subsection{Central and exponential central gauges on harmonic spaces}

A gauge $\varphi=\left(\varphi^{\mathcal{V}}, \varphi^{G}\right): P \mid \mathcal{V} \rightarrow \mathcal{V} \times G$ for the $G$-bundle $P$ naturally corresponds to a gauge for its lift $P^{\prime}$ on the harmonic space $\mathcal{H}(M)$, namely the gauge $\varphi$ defined on the restriction of $P^{\prime}$ to $\mathcal{V} \times \mathrm{SL}_{2}(\mathbb{C}) \subset \mathcal{H}(M)$ by

$$
\varphi: P^{\prime} \mid \mathcal{V} \times \mathrm{SL}_{2}(\mathbb{C}) \rightarrow \mathcal{V} \times \mathrm{SL}_{2}(\mathbb{C}) \times G, \quad \varphi(u, U):=\left(\varphi^{\mathcal{V}}(u), U, \varphi^{G}(u)\right) .
$$

Such a gauge is called the central gauge determined by $\varphi[1,24]$.

Let us now introduce a very convenient special class of central gauges. Given $x_{o} \in M$, for any unit vector $v \in T_{x_{o}} M$ we denote by $\gamma_{t}^{(v)}=\exp _{x_{o}}(t v)$ the radial geodesic determined by $v$. Now let $\mathcal{V} \subset T_{x_{o}} M$ be a neighbourhood of $x_{o}$ such that the exponential map $\exp _{x_{o}}: \mathcal{V} \subset$ $T_{x_{o}} M \rightarrow M$ is a diffeomorphism onto its image $\mathcal{U}=\exp _{x_{o}}(\mathcal{V})$. A gauge $\varphi: P \mid u \rightarrow U \times G$ on the domain $U=\exp (\mathcal{V})$ is called exponential if the corresponding potential $A$ satisfies the following conditions:

(a) $\left.A\right|_{x_{o}}=0$ and

(b) $A\left(\dot{\gamma}_{t}^{(v)}\right)=0$ for all vectors $\dot{\gamma}_{t}^{(v)}$ tangent to the radial geodesics.

We shall call the central gauges for $P^{\prime}$ on $\mathcal{H}(M)$ determined by exponential gauges for $P$ on $M$ exponential central (or just exp-central). We recall that for any $x_{o} \in M$ there is always an exponential gauge on some appropriate neighbourhood of $x_{o}$ [37]. Thus, for any $\left(x_{o}, U\right) \in \mathcal{H}(M)$ there exists an exp-central gauge for $P^{\prime}$ on a neighbourhood of $\left(x_{o}, U\right)$.

It is also known that for all cases in which $(E, D)$ is the complexification of a gauge field $\left(E^{o}, D^{o}\right.$ ) with compact structure group $G^{o} \subset G$, if $A$ is the potential in an exponential gauge $\varphi$ for the bundle $P^{o}$ with domain $\mathcal{U} \subset M$, then there exists a constant $c \mathcal{U}$, which depends only on $\mathcal{U}$, such that

$$
\|A\|_{\mathcal{C}^{0}(K, \mathfrak{g})} \leq c \mathcal{U}\|F\|_{\mathcal{C}^{0}(K, \mathfrak{g})}^{(\varphi)} \text { for } K \subset \subset \mathcal{U},
$$

(see, for example, [37, Lemma 2.1]). Similar estimates clearly hold for potentials and curvatures of the lifted gauge fields $\left(E^{\prime}, D^{\prime}\right)$ in exp-central gauges.

\subsection{Prepotentials for instantons on hk manifolds}

We recall that any $h k$ manifold $\left(M, g,\left(J_{\alpha}\right)\right)$ has a natural structure of a real analytic manifold and that in such a structure, the tensors $g$ and $J_{\alpha}$ are real analytic [26]. Hence, if we lift $g$ to $\mathcal{H}(M)$ as a tensor field with values in $\mathcal{D}^{*} \otimes \mathcal{D}^{*}$, using real analyticity, for each point $(x, U) \in \mathcal{H}(M) \subset \mathcal{H}^{\mathbb{C}}(M)$ we may determine a tubular $\mathrm{SL}_{2}(\mathbb{C})$-invariant neighbourhood $\mathcal{W} \subset \mathcal{H}^{\mathbb{C}}(M)$ of $(x, U)$, to which $g$ extends as a $\mathbb{C}$-linear tensor field in $\mathcal{D}^{\mathbb{C} *} \times \mathcal{D}^{\mathbb{C} *}$.

We claim that an analogous extension property holds also for an instanton on an hk manifold provided that it is a complexification of an instanton with compact structure group. To prove this, let us introduce some additional convenient notation. Given a gauge field $(E, D)$ associated with $(P, \omega)$, let $\mathfrak{H} \subset T P^{\prime}$ and $\mathfrak{H}^{\mathbb{C}} \subset T^{\mathbb{C}} P^{\prime}$ be, respectively, the real and complex horizontal distributions of $P^{\prime}$ given by the kernels of the lifted connection $\omega^{\prime}$ on $P^{\prime}$. Further, for any (real or complex) vector field $X$ on $\mathcal{H}(M)$, let us denote by $X^{h}$ the uniquely associated vector field on $P^{\prime}$ with values in $\mathfrak{H}$ or $\mathfrak{H}^{\mathbb{C}}$ which projects onto $X$.

Proposition 4.1 If $(E, D)$ is an instanton on $\left(M, g,\left(J_{\alpha}\right)\right)$, which is the complexification of a gauge field with compact structure group $G^{o}$, all data of the lifted pairs $\left(E^{\prime}, D^{\prime}\right)$ and $\left(P^{\prime}, \omega^{\prime}\right)$ on $\mathcal{H}(M)$ are real analytic. Moreover, there is a complex structure $J^{\prime}$ on $P^{\prime}$, invariant 
under $G=\left(G^{o}\right)^{\mathbb{C}}$, which makes $p^{\prime}: P^{\prime} \rightarrow \mathcal{H}(M)$ and the associated vector bundle $p^{E^{\prime}}: E^{\prime} \rightarrow \mathcal{H}(M)$ holomorphic bundles over $\mathcal{H}(M)$ with $J^{\prime}$-invariant connections.

It follows that, for any $\left(x_{o}, U\right) \in \mathcal{H}(M) \subset \mathcal{H}^{\mathbb{C}}(M)$, there are unique real analytic extensions of $\left(E^{\prime}, D^{\prime}\right)$ and $\left(P^{\prime}, \omega^{\prime}\right)$ to some $\mathrm{SL}_{2}(\mathbb{C})$-invariant tubular neighbourhood $\mathcal{W} \subset \mathcal{H}^{\mathbb{C}}(M)$ of the real submanifold $\mathcal{U}:=\mathcal{W} \cap \mathcal{H}(M)$ containing $\left(x_{o}, U\right)$. Further, both the extended bundles $E^{\prime}, P^{\prime}$ have naturally extended complex structures that make them holomorphic bundles over $\left(\mathcal{H}^{\mathbb{C}}(M), \mathbb{I}^{(\mathbb{C})}\right)$ and the extended connection $\omega^{\prime}$ is $J^{\prime \mathbb{C}}$-invariant with respect to the complex structure $J^{\prime \mathbb{C}}$ of $P^{\prime}$.

Finally, the components in holomorphic gauges of the extended connection $\omega^{\prime}$ and of its $(1,0)$-potential are holomorphic functions of any set of complex coordinates $\left(z^{\ell}, w^{m},\left(u_{r}^{i}\right)\right)$ of the complex manifold $\left(\mathcal{H}^{\mathbb{C}}(M)=M \times M \times \mathrm{SL}_{2}(\mathbb{C}), \mathbb{I}^{\mathbb{C}}\right)$ that correspond to complex coordinates $\left(z^{\ell}, \bar{z}^{m}=w^{m},\left(u_{r}^{i}\right)\right)$ of the submanifold $\mathcal{H}(M) \subset \mathcal{H}^{\mathbb{C}}(M)$,

Proof To prove these statements, we need the following simple lemma.

Lemma 4.2 The complex gauge field $(E, D)$ is an instanton if and only if the curvature $F^{\prime}$ of its lift $\left(E^{\prime}, D^{\prime}\right)$ on $\mathcal{H}(M)$ is such that

$$
F^{\prime}\left(X_{+}, Y_{+}\right)=0=F^{\prime}\left(\overline{X_{+}}, \overline{Y_{+}}\right) \text {for } X_{+}, Y_{+} \in \mathcal{D}_{+} .
$$

Proof of Lemma. As observed above, each space $\left.\mathcal{D}_{+}\right|_{(x, U)}$ or (more precisely, its isomorphic projection onto $T_{x}^{\mathbb{C}} M$ ) is spanned by the vectors $e_{+a}$ of the adapted frames $u: \mathbb{H}^{n} \rightarrow$ $T_{x} M$. Hence, the curvature of $(E, D)$ satisfies the first condition in (4.2) if and only if $F^{\prime}\left(X_{+}, Y_{+}\right)=0$ and $F^{\prime}\left(X_{-}, Y_{-}\right)=0$ for $X_{ \pm}, Y_{ \pm} \in \mathcal{D}_{ \pm}$. The other conditions in (4.2) are direct consequences of the decomposition of $\left.\mathcal{D}^{\mathbb{C}}\right|_{(x, U)} \simeq T_{x}^{\mathbb{C}} M$ into irreducible $\mathrm{SU}_{2}$-moduli.

Due to this lemma and (2.4), the curvature 2-form $\Omega^{\prime}$ of the lifted connection $\omega^{\prime}$ identically vanishes on any pair of horizontal lifts $X^{01 h}, Y^{01 h} \in \mathfrak{H}^{\mathbb{C}}$ of vector fields $X^{01}, Y^{01}$ in the antiholomorphic distribution $\mathcal{D}_{-}+V^{01}=T^{01} \mathcal{H}(M)$ of $(\mathcal{H}(M), \mathbb{I})$, where $\mathcal{D}_{-}$and $V^{01}$ are the $\mathbb{I}$-anti-holomorphic horizontal and vertical distributions of $\mathcal{H}(M)$ described in Sect. 3.2. It follows that the subbundle $\mathcal{S}^{01} \subset T^{\mathbb{C}} P^{\prime}$ generated by the vectors $X^{01 h}$ and the antiholomorphic vertical distribution of $P^{\prime}$ is an involutive complex distribution. The same holds for the subbundle $\mathcal{S}^{10}=\overline{\mathcal{S}^{01}} \subset T^{\mathbb{C}} P^{\prime}$. Hence, the direct sum decomposition $T^{\mathbb{C}} P^{\prime}=$ $\mathcal{S}^{10}+\mathcal{S}^{01}$ corresponds to a $G$-invariant integrable complex structure $J^{\prime}$ on $P^{\prime}$. Consequently, there is an atlas of complex charts for the complex manifold $\left(P^{\prime}, J^{\prime}\right)$ making $P^{\prime}$ a holomorphic $G$-bundle over $\mathcal{H}(M)$. Moreover, the lift $P^{o \prime}$ on $\mathcal{H}(M)$ of the bundle $P^{o}$ with compact structure group is necessarily a real analytic submanifold of $P^{\prime}$ since it is the fixed point set of an appropriate real analytic involution. One can also check that the restricted distribution $\left.\mathfrak{H}\right|_{P^{\prime \prime}}$ coincides with the distribution given by the $J^{\prime}$-invariant subspaces of the tangent spaces of $P^{o \prime}$. Since the latter is a real analytic distribution on $P^{o \prime}$ and the distribution $\mathfrak{H}$ of $P^{\prime}$ is the unique $G$-invariant extension of $\left.\mathfrak{H}\right|_{P^{o \prime}}$, we conclude that also $\mathfrak{H}$ is $J^{\prime}$-invariant on $P^{\prime}$. Consequently, the first claim follows immediately.

Concerning the second claim, the same arguments as above yield the existence of a complex structure $J^{\prime \mathbb{C}}$ on the extended bundle $P^{\prime}$ (and, consequently, a corresponding complex structure on the associated bundle $E^{\prime}$ ), which makes it a holomorphic bundle over the complex manifold $\left(\mathcal{H}^{\mathbb{C}}(M), \mathbb{I}^{(\mathbb{C})}\right)$ and leaves invariant the horizontal distribution $\mathfrak{H}$ determined by the extended connection 1 -form $\omega^{\prime}$. The final claim is a consequence of the fact that the extension to $\mathcal{W} \subset \mathcal{H}^{\mathbb{C}}(M)$ of every real analytic datum on $\mathcal{H}(M)$ is obtained by considering the power series of such a datum in the variables $\left(z^{\ell}, w^{m}:=\bar{z}^{m},\left(u_{\ell}^{i}\right)\right)$ as a power series in 
the independent complex variables $\left(z^{\ell}, w^{m},\left(u_{\ell}^{i}\right)\right)$, thus holomorphic in both the variables $z^{\ell}$ and $w^{m}$. The holomorphy in the $u_{\ell}^{i}$ follows from the fact that $\omega^{\prime}$ is the lift of a connection form of $p: P \rightarrow M$ to the bundle $p^{\prime}: P^{\prime} \rightarrow \mathcal{H}(M)$.

Consider the basis $\left(H_{0}^{o}, H_{++}^{o}, H_{--}^{o}\right)$ of $\mathfrak{s l}_{2}(\mathbb{C})$ defined by

$$
H_{0}^{o}:=\left(\begin{array}{cc}
1 & 0 \\
0 & -1
\end{array}\right), \quad H_{++}^{o}:=\left(\begin{array}{cc}
0 & 1 \\
0 & 0
\end{array}\right), \quad H_{--}^{o}:=\left(\begin{array}{cc}
0 & 0 \\
1 & 0
\end{array}\right)
$$

and denote by $H_{0}, H_{++}, H_{--}$the associated holomorphic vector fields on $\mathcal{H}(M)$, determined as holomorphic parts of the infinitesimal transformations of the right actions of the oneparameter groups generated by $H_{0}^{o}, H_{++}^{o}, H_{--}^{o}$. The restrictions of such vector fields $H_{\alpha}$ to each vertical fibre $\{x\} \times \mathrm{SL}_{2}(\mathbb{C}) \simeq \mathrm{SL}_{2}(\mathbb{C})$ are left invariant and generate a Lie algebra isomorphic to $\mathfrak{s l}_{2}(\mathbb{C})$.

We may now prove the theorem, on which all our results are based (see also [1, Prop. 7 and Thm. 4]).

Theorem 4.3 Given a real analytic complex instanton $(E, D)$ and a point $\left(x_{o}, U\right) \in \mathcal{H}(M)$, there exists an $\mathrm{SL}_{2}(\mathbb{C})$-invariant neighbourhood $\mathcal{U} \subset \mathcal{H}^{\mathbb{C}}(M)$ of $\left(x_{o}, x_{o}, U\right)$ and a holomorphic gauge $\varphi: P^{\prime} \mid u \rightarrow \mathcal{U} \times G$ of the $\mathcal{H}^{\mathbb{C}}(M)$-extension of the lifted bundle of $P$ with associated (1,0)-potential A satisfying the conditions

$$
A\left(H_{0}\right)=A\left(X_{-}\right)=0, \quad X_{-} \in \mathcal{D}_{-} .
$$

Such a potential $A$ is uniquely determined by the $\mathfrak{g}$-valued function $A_{--}:=A\left(H_{--}\right)$in the following sense: given the lift $\left(\widehat{E}^{\prime}, \widehat{D}^{\prime}\right)$ on $\mathcal{H}(M)$ of a real analytic complex instanton $(\widehat{E}, \widehat{D})$ on $M$, if $\widehat{E}^{\prime} \mid u$ coincides with $E^{\prime} \mid u$ and if furthermore the $(1,0)$-potential $\widehat{A}$ of $\left(\widehat{E}^{\prime}, \widehat{D}^{\prime}\right)$ in a holomorphic gauge satisfies (4.6), then

$$
\widehat{D}^{\prime}\left|u \cap \mathcal{H}(M)=D^{\prime}\right| u \cap \mathcal{H}(M) \Longleftrightarrow \widehat{A}\left(H_{--}\right)\left|u \cap \mathcal{H}(M)=A\left(H_{--}\right)\right| u \cap \mathcal{H}(M) .
$$

Remark 4.4 Note that by $\widehat{D}^{\prime}\left|u \cap \mathcal{H}(M)=D^{\prime}\right| u \cap \mathcal{H}(M)$ we mean that the differential operators $D^{\prime}, \widehat{D}^{\prime}$ are identical, not just equivalent up to an automorphism (gauge transformation) of $\widehat{E}^{\prime} \mid$ u. However, (4.7) implies a bijective correspondence between covariant derivatives and their potential components $A_{--}=A\left(H_{--}\right)$. This induces a natural bijective correspondence between equivalence classes of instantons up to local automorphisms and equivalence classes of $(1,0)$-potential components $A_{--}=A\left(H_{--}\right)$up to appropriate gauge transformations (2.2). In fact, the required gauge transformations are precisely those leaving condition (4.6) unchanged.

Proof Let us extend $\left(P^{\prime}, \omega^{\prime}\right)$ to an $\mathrm{SL}_{2}(\mathbb{C})$-invariant tubular neighbourhood $\mathcal{W} \subset \mathcal{H}^{\mathbb{C}}(M)$ of $\mathcal{H}(M)$ and consider the two $G$-invariant complex distributions $\mathcal{D}_{-}^{h}$ and $\mathcal{D}_{-}^{h} \oplus<H_{0}^{h}>$ on the extended bundle $P^{\prime}$, the former generated by the horizontal lifts $X^{h}$ of the complex vector fields in $\mathcal{D}_{-}$, the latter generated by the complex vector fields in $\mathcal{D}_{-}^{h}$ and the horizontal lift $H_{0}^{h}$ of the holomorphic vector field $H_{0}$ of $\mathcal{H}^{\mathbb{C}}(M)$. We recall that the distribution $\mathcal{D}_{-}$is spanned by the complex vector fields $e_{-a}$ described in Sect. 2.1. Using this and the identification, described in Remark 3.1, between the points of $\mathcal{H}(M)$ and the frames for the fibres $\mathbf{H}_{x}$ of the bundle $\mathbf{H}$, the distribution $\mathcal{D}_{-}$is seen to be invariant under the flow of the vector field $H_{0}$. This implies that the horizontal distribution $\mathcal{D}_{-}^{h}$ is invariant under the flow of the horizontal vector field $H_{0}^{h}$.

By Lemma 4.2, the curvature 2-form $\Omega^{\prime}$ of $\omega^{\prime}$ vanishes identically on the distribution $\mathcal{D}_{-}^{h}$, meaning that $\mathcal{D}_{-}^{h}$ is involutive. Moreover, from the last claim in Proposition $4.1, \mathcal{D}_{-}^{h}$ is generated by holomorphic vector fields of the holomorphic bundle $\pi: P^{\prime} \rightarrow \mathcal{W} \subset \mathcal{H}^{\mathbb{C}}(M)$. 
Hence, by the complex Frobenius theorem, for each $y_{o}=\left.\left(x_{o}, x_{o}, U, g\right) \in P^{\prime}\right|_{\mathcal{H}(M)}$ there is an $\mathrm{SL}_{2}(\mathbb{C}) \times G$-invariant neighbourhood $P^{\prime} \mid u$ of $y_{o}$, which is holomorphically foliated by integral leaves of $\mathcal{D}_{-}^{h}$. Note that the union of the $H_{0}^{h}$-orbits of the points of one such integral leaf is an integral leaf of the larger complex distribution $\mathcal{D}_{-}^{h} \oplus<H_{0}^{h}>$. It follows that $P^{\prime} \mid u$ is actually holomorphically foliated by the integral leaves of this larger distribution and we may consider a holomorphically parameterised family of integral leaves of this distribution, which fills a complex submanifold $\mathcal{S}^{\prime}$ transversal to the $G$-orbits at each point. Without loss of generality, we may also assume that such a submanifold $\mathcal{S}^{\prime}$ projects biholomorphically onto an $\mathrm{SL}_{2}(\mathbb{C})$-invariant neighbourhood $\mathcal{U} \subset \mathcal{H}^{\mathbb{C}}(M)$ of $\left(x_{o}, x_{o}, U\right)$ and hence it is a graph of a holomorphic section of the $G$-bundle $P^{\prime}$. Associated with such a section, there is a unique holomorphic gauge $\varphi: P^{\prime} \mid \mathfrak{U} \rightarrow \mathcal{U} \times G$ mapping $\mathcal{S}^{\prime}$ onto the trivial section $\mathcal{U} \times\{e\}$ of the trivial bundle $\mathcal{U} \times G$. By construction, the (1,0)-potential $A$ in such a gauge satisfies (4.6).

Suppose now that $\left(\widehat{E}^{\prime}, \widehat{D}^{\prime}\right)$ is a real analytic lifted instanton associated with the pair $\left(\widehat{P}^{\prime}, \widehat{\omega}^{\prime}\right)$, and assume that $\widehat{A}\left(H_{--}\right)\left|u \cap \mathcal{H}(M)=A\left(H_{--}\right)\right| u \cap \mathcal{H}(M)$. By real analyticity, we may assume that $\mathcal{U}$ is such that $\widehat{A}\left(H_{--}\right)\left|u=A\left(H_{--}\right)\right| u$. Thus, the horizontal lifts $H_{\alpha}^{h}, \widehat{H}_{\alpha}^{h}$ of the holomorphic vector fields $H_{\alpha}, \alpha \in\{0,++,--\}$, determined by the connection forms $\omega^{\prime}$ and $\widehat{\omega}^{\prime}$, respectively, have the forms

$$
\begin{aligned}
& \widehat{H}_{0}^{h}=H_{0}^{h}, \quad \widehat{H}_{--}^{h}=H_{--}+\widehat{A}_{--}=H_{--}+A_{--}=H_{--}^{h}, \\
& \widehat{H}_{++}^{h}=H_{++}+\widehat{A}_{++}, \quad H_{++}^{h}=H_{++}+A_{++},
\end{aligned}
$$

where we denote by $A_{ \pm \pm}:=A\left(H_{ \pm \pm}\right), \widehat{A}_{ \pm \pm}:=\widehat{A}\left(H_{ \pm \pm}\right)$the components along the vector fields $H_{ \pm \pm}$of the corresponding $(1,0)$-potentials. Setting $B_{++}:=\widehat{A}_{++}-A_{++}$, the expansions (4.8) can be written as

$$
\widehat{H}_{0}^{h}=H_{0}^{h}, \quad \widehat{H}_{++}^{h}=H_{++}^{h}+B_{++}, \quad \widehat{H}_{--}^{h}=H_{--}^{h} .
$$

Since the considered principal bundles $P^{\prime}, \widehat{P}^{\prime}$ are lifts to $\mathcal{H}(M)$ of bundles over $M$, the Lie brackets among the horizontal lifts of vector fields $H_{A}$ coincide with the Lie brackets among their projections (see (2.10)) so that

$$
\left[\widehat{H}_{0}^{h}, \widehat{H}_{++}^{h}\right]=2 \widehat{H}_{++}^{h}, \quad\left[\widehat{H}_{++}^{h}, \widehat{H}_{--}^{h}\right]=\widehat{H}_{0}^{h} .
$$

This and (4.9) imply that the $\mathfrak{g}$-valued holomorphic function $B_{++}$is such that

$$
H_{0}^{h} \cdot B_{++}=2 B_{++}, \quad H_{--}^{h} \cdot B_{++}=0 .
$$

On the other hand, by Proposition 2.2, the vector fields $H_{0}^{h}, H_{++}^{h}, H_{--}^{h}$ generate a right holomorphic $\mathrm{SL}_{2}(\mathbb{C})$-action on $P^{\prime} \mid u$ and the bundle $P^{\prime} \mid u$ is foliated by regular orbits of this action. (Thus, each such orbit is identifiable with a copy of $\mathrm{SL}_{2}(\mathbb{C})$.) We may therefore apply Lemma 5.3 in [16] (see also [24, Sect. 4.3]) - considered for the pair of vector fields $H_{0}$, $H_{--}$in place of the pair $H_{0}, H_{++}$- to the restrictions of $B_{++}$to each such orbit. This lemma implies that $B_{++}$is identically vanishing along each such orbit. It follows that $B_{++} \equiv 0$, i.e. $\widehat{A}_{++} \equiv A_{++}$, and that $\widehat{H}_{++}^{h}=H_{++}^{h}$.

In order to conclude that $\widehat{D}^{\prime}=D^{\prime}$, it is now sufficient to prove the existence of a collection of holomorphic vector fields $\left(e_{+a}, e_{-b}\right)$ on $\mathcal{U} \subset \mathcal{H}^{\mathbb{C}}(M)$ with the following two properties: a) they span a distribution which is complementary to the one generated by the vector fields $H_{A}$ and b) the remaining components $\widehat{A}_{ \pm a}:=A\left(e_{ \pm a}\right)$ and $A_{ \pm a}=A\left(e_{ \pm a}\right)$ of the potentials of $\widehat{D}^{\prime}$ and $D^{\prime}$ are identical. Let us consider a set of (locally defined) holomorphic vector fields $\left(e_{-a}\right)$ generating the distribution $\mathcal{D}_{-}$and projecting pointwise onto vectors $e_{-a} \in T^{\mathbb{C}} M$ determined by adapted frames as in Sect. 2.1. Since the vector fields $e_{-a}$ are eigenvectors with eigenvalue -1 for the action of the complex vector field $H_{0}$, the vectors $e_{+a}:=\left[H_{++}, e_{-a}\right]$ 
are eigenvectors with eigenvalue +1 for the same action. This implies that the $e_{+a}$ generate the distribution $\mathcal{D}_{+}$and that $\left(e_{+a}, e_{-b}\right)$ is a collection of generators for the distribution $\mathcal{D}_{\mathbb{T}}^{10}$ complementary to the distribution spanned by the $H_{A}$. Moreover, their corresponding lifts $e_{ \pm a}^{h}, \widehat{e}_{ \pm a}^{h}$, determined by the two connection forms $\omega^{\prime}, \widehat{\omega}^{\prime}$, are such that

$$
\left[H_{++}^{h}, e_{-a}^{h}\right]=e_{+a}^{h}, \quad\left[\widehat{H}_{++}^{h}, \quad \widehat{e}_{-a}^{h}\right]=\widehat{e}_{+a}^{h} .
$$

Setting $A_{ \pm a}:=A\left(e_{ \pm a}\right), \widehat{A}_{ \pm a}:=\widehat{A}\left(e_{ \pm a}\right)$ and recalling that, by hypothesis, $A_{-a}=\widehat{A}_{-a}=0$, we have that (4.10) implies $-e_{-a}^{h} \cdot A_{++}=A_{+a}$ and $-e_{-a}^{h} \cdot \widehat{A}_{++}=A_{+a}$. Since we have proven that $\widehat{A}_{++} \equiv A_{++}$, this gives $\widehat{A}_{ \pm a} \equiv A_{ \pm a}$, as desired.

We call the $\mathfrak{g}$-valued map $A_{--} \mid u \cap \mathcal{H}(M):=A\left(H_{--} \mid u \cap \mathcal{H}(M)\right)$, which uniquely determines the extended holomorphic function $A_{--} \mid u$ and thereby the gauge field $\left(E^{\prime} \mid u, D^{\prime}\right)$, a prepotential on $\mathcal{U} \cap \mathcal{H}(M)$ for the instanton $(E, D)$. The holomorphic gauges of the (extended) lifted bundle $P^{\prime}$ in which the potential of $\omega^{\prime}$ satisfies (4.6) are called analytic.

Remark 4.5 The previous literature on the harmonic space formulation, e.g. [1,22-24], used gauge conditions $A_{0}=A_{+a}=0$ and prepotentials $A_{++}$. Here we choose to reverse the role of the signs. This has the advantage that prepotentials are holomorphic rather than antiholomorphic with respect to the complex structure $\mathbb{I}$ of $\mathcal{H}(M)$ (see Remark 4.7).

\subsection{Analytic gauges, bridges and normalisations}

Assume that our instanton $(E, D)$ is the complexification of an instanton with compact structure group $G^{o}$ over the hk manifold $\left(M, g,\left(J_{\alpha}\right)\right)$. Around each $\left(x_{o}, U\right) \in \mathcal{H}(M)$ there are two very important classes of gauges to be considered: the exp-central gauges and the analytic gauges. Let us briefly compare their main features:

- If $\varphi: P^{\prime} \mid \nu_{\times \mathrm{SL}_{2}(\mathbb{C})} \rightarrow \mathcal{V} \times \mathrm{SL}_{2}(\mathbb{C}) \times G$ is (the restriction to $\mathcal{H}(M) \subset \mathcal{H}^{\mathbb{C}}(M)$ of) an analytic gauge, the corresponding holomorphic potential $A$ for $\omega$ is such that $A_{0}:=$ $A\left(H_{0}\right)$ is identically vanishing and $A\left(X_{-}\right)=0$ for any vector field $X_{-} \in \mathcal{D}_{-}$. In contrast with this, the functions $A_{ \pm \pm}:=A\left(H_{ \pm \pm}\right)$and the functions $A\left(X_{+}\right), X_{+} \in \mathcal{D}_{+}$, are in general non-trivial.

- If $\tilde{\varphi}: P^{\prime} \mid \mathcal{} \times \mathrm{SL}_{2}(\mathbb{C}) \rightarrow \mathcal{V} \times \mathrm{SL}_{2}(\mathbb{C}) \times G$ is an exp-central gauge, the corresponding potential $\widetilde{A}$ is such that the components $\widetilde{A}_{\alpha}=\widetilde{A}\left(H_{\alpha}\right), \alpha \in\{0,++,--\}$, identically vanish, while all functions $\widetilde{A}\left(X_{ \pm}\right), X_{ \pm} \in \mathcal{D}_{ \pm}$, are in general non-trivial, being nevertheless constrained by the conditions (a), (b) in Sect. 4.2.

The maps $g: \mathcal{U} \times \mathrm{SL}_{2}(\mathbb{C}) \rightarrow G$, which give the gauge transformations $h \rightarrow g_{(x, U)} \cdot h$ from central gauges to analytic gauges are usually called bridges (e.g. $[1,23,24])$. Now, since $G$ is the complexification of the compact Lie group $G^{o}$, it is reductive and the exponential $e^{(\cdot)}: \mathfrak{g} \rightarrow G$ is a surjective local diffeomorphism. This means that any bridge $g_{(x, U)}$ can be written as $g_{(x, U)}=e^{\psi_{(x, U)}}$ for some appropriate $\mathfrak{g}$-valued function $\psi$. We call $\psi$ a $\mathfrak{g}$-bridge.

In the next lemma we shall give the proof of existence of bridges and $\mathfrak{g}$-bridges, having the special property that the prepotentials determined in the newly built analytic gauges satisfy additional normalisation conditions, which drastically reduce their degrees of freedom. Such normalised analytic gauges can be considered as complex analogues of the Coulomb gauges for arbitrary gauge fields.

In order to properly state such a normalisation, we need to introduce some appropriate notation. Given $x_{o} \in M$ and a (sufficiently small) simply connected neighbourhood $\mathcal{V} \subset M$ of $x_{o}$, let $\left(e_{+a}, e_{-b}\right)$ be a $4 n$-tuple of holomorphic vector fields of $\mathcal{H}^{\mathbb{C}}(M)$ that generate 
the distributions $\mathcal{D}_{ \pm} \subset T^{\mathbb{C}}\left(\mathcal{V} \times \mathcal{V} \times \mathrm{SL}_{2}(\mathbb{C})\right)\left(\subset T^{\mathbb{C}} \mathcal{H}^{\mathbb{C}}(M)\right)$, constructed as in the proof of Theorem 4.3. Then, pick an element $U_{o} \in \mathrm{SL}_{2}(\mathbb{C})$, say $U_{o}=\left(\begin{array}{ll}1 & 0 \\ 0 & 1\end{array}\right)$, and let $\lambda^{a}, \mu$ : $\mathcal{V} \times \mathcal{V} \times \mathrm{SL}_{2}(\mathbb{C}) \rightarrow \mathbb{C}^{2 n}, a=1, \ldots, 2 n$, be a set of $2 n+1$ holomorphic functions satisfying the following conditions

$$
\begin{aligned}
& H_{++} \cdot \lambda^{a}=0, \quad H_{++} \cdot \mu=-1, \quad H_{0} \cdot \lambda^{a}=H_{0} \cdot \mu=0, \\
& e_{+b} \cdot \lambda^{a}=\delta_{b}^{a}, \quad e_{+b} \cdot \mu=0,\left.\quad \lambda^{a}\right|_{\left(x_{o}, x_{o}, U_{o}\right)}=\left.\mu\right|_{\left(x_{o}, x_{o}, U_{o}\right)}=0 .
\end{aligned}
$$

By [16, Lemma 5.2], functions satisfying the first line of these conditions surely exist and are determined up to addition of a holomorphic function constant along each $\mathrm{SL}_{2}(\mathbb{C})$-orbits. Using the commutation relations between $H_{++}, H_{0}$ and $e_{+a}$, we can see that also the second line of these conditions can be satisfied, fixing the $\lambda^{a}$ and $\mu$ completely. Now consider the holomorphic distribution $\widetilde{\mathcal{D}} \subset T^{\mathbb{C}}\left(\mathcal{V} \times \mathcal{V} \times \mathrm{SL}_{2}(\mathbb{C})\right)$ generated by the vector fields

$$
e_{+a}, \quad H_{++}, \quad \widetilde{H}_{--}:=H_{--}+\lambda^{a} e_{-a}+\mu H_{0},
$$

which can be directly checked to be involutive. Finally let $\widehat{S}$ and $S$ be two complex submanifolds of $\mathcal{V} \times \mathcal{V} \times \mathrm{SL}_{2}(\mathbb{C}) \subset \mathcal{H}^{\mathbb{C}}(M)$, one included in the other, which are integral leaves of the holomorphic distributions $\mathcal{D}_{+}$and $\widetilde{\mathcal{D}}$, respectively, and both passing through $\left(x_{o}, x_{o}, U_{o}\right)$. Note that each tangent space of $S$ is complementary to $\left.\mathcal{D}_{-}\right|_{(x, y, U)}+<\left.H_{0}\right|_{(x, y, U)}>$.

We may now state and prove the advertised existence result.

Lemma 4.6 Let $\tilde{\varphi}: P^{\prime} \mid \mathcal{V} \times \mathrm{SL}_{2}(\mathbb{C}) \rightarrow \mathcal{V} \times \mathrm{SL}_{2}(\mathbb{C}) \times G$ be an exp-central gauge, determined by an exponential gauge for $(P, \omega)$ on a simply connected neighbourhood $\mathcal{V} \subset M$ around $x_{o} \in M$, and $\widetilde{A}$ the associated potential for $\omega$. Let also $\widehat{S} \subset S \subset \mathcal{V} \times \mathcal{V} \times \mathrm{SL}_{2}(\mathbb{C})$ be the pair of complex submanifolds passing through $\left(x_{o}, U_{o}\right)$ described above. If $\mathcal{V}$ is sufficiently small, there exists an analytic gauge $\varphi: P^{\prime} \mid \mathcal{V} \times \mathcal{V} \times \mathrm{SL}_{2}(\mathbb{C}) \rightarrow \mathcal{V} \times \mathcal{V} \times \mathrm{SL}_{2}(\mathbb{C}) \times G$, in which the prepotential $A_{--}$is such that

$$
\begin{aligned}
H_{0} \cdot A_{--} & =-2 A_{--}, \quad e_{-a} \cdot A_{--}=0, \quad H_{--} \cdot A_{--}=0, \\
A_{--} \mid \widehat{S} & =-\lambda^{a} \widetilde{A}_{-a}\left|\widehat{S}, \quad H_{++} \cdot A_{--}\right| \widehat{S}=-\lambda^{a} \widetilde{A}_{+a} \mid \widehat{S} .
\end{aligned}
$$

Proof For simplicity, we use $\tilde{\varphi}$ to identify $P^{\prime} \mid \mathcal{V} \times \mathrm{SL}_{2}(\mathbb{C})$ with $\mathcal{V} \times \mathrm{SL}_{2}(\mathbb{C}) \times G$ so that we may assume that the considered exp-central gauge is nothing but the identity map. Let us consider the integral leaves in $\mathcal{V} \times \mathcal{V} \times \mathrm{SL}_{2}(\mathbb{C}) \times G$ of the holomorphic distribution $\mathcal{D}_{-}^{h}+<H_{0}^{h}>$, which passes through the points of the manifold $S \times\{e\} \subset \mathcal{V} \times \mathcal{V} \times \mathrm{SL}_{2}(\mathbb{C}) \times\{e\}$. Being horizontal, they are transversal to the $G$-orbits and, by dimension counting, they fill a submanifold $\mathcal{S}^{\prime} \subset P^{\prime}$ which projects diffeomorphically onto an $\mathrm{SL}_{2}(\mathbb{C})$-invariant neighbourhood $\mathcal{V}^{\prime} \times \mathcal{V}^{\prime} \times \mathrm{SL}_{2}(\mathbb{C})$ of $\left(x_{o}, x_{o}, U_{o}\right)$. Thus, there is a gauge $\varphi^{o}: P^{\prime} \mid \mathcal{V} \times \mathcal{V} \times \mathrm{SL}_{2}(\mathbb{C}) \rightarrow$ $\mathcal{V} \times \mathcal{V} \times \mathrm{SL}_{2}(\mathbb{C}) \times G$, which maps $\mathcal{S}^{\prime}$ into the submanifold $\mathcal{V} \times \mathcal{V} \times \mathrm{SL}_{2}(\mathbb{C}) \times\{e\}$ and which we may assume to satisfy the condition $\left.\varphi^{o}\right|_{S \times\{e\}}=\operatorname{Id}_{S \times\{e\}}$ (see also the proof of Theorem 4.3). By construction, $\varphi^{o}$ is an analytic gauge and the bridge $g_{(x, y, U)}$ from $\tilde{\varphi}=\operatorname{Id}$ to $\varphi^{o}$ is such that $h \mapsto g_{(x, y, U)}^{o} \cdot h=e \cdot h$ for each $(x, y, U) \in S$. Hence, writing this bridge in the form $g_{(x, y, U)}^{o}=e^{\psi^{o}(x, y, U)}$ for an appropriate $\mathfrak{g}$-bridge $\psi^{o}$, we have that $\left.\psi^{o}\right|_{S} \equiv 0$. Note that $\psi^{o}$ satisfies the equations $H_{0} \cdot \psi^{o}=0$ and $e_{-a} \cdot \psi^{o}+\widetilde{A}\left(e_{-a}\right)=0$ because of the following three properties: (a) in any central gauge the potential $\widetilde{A}$ satisfies $\widetilde{A}\left(H_{0}\right)=0$, (b) the potential $A^{o}$ in the analytic gauge $\varphi^{o}$ satisfies (4.6) and (c) the potentials $\widetilde{A}$ and $A^{o}$ are related by (2.2).

Let us now denote by $A_{--}^{o}$ the prepotential of the given instanton in this gauge $\varphi^{o}$. Expanding the identities $\mathcal{F}^{\varphi_{o}}\left(H_{0}, H_{--}\right)=\mathcal{F}^{\varphi_{o}}\left(e_{-a}, H_{--}\right)=0$ in terms of the potential $A^{o}$ in this gauge and recalling that, being an analytic gauge, we have $A_{0}^{o}=A_{-a}^{o}=0$, we find 
that $H_{0} \cdot A_{--}^{o}=-2 A_{--}^{o}$ and $e_{-a} \cdot A_{--}^{o}=0$, i.e. the first two conditions of (4.12). We remark that these two conditions are satisfied by any prepotential, being merely consequences of the above properties of the curvature.

However, the prepotential $A_{--}^{o}$ does not necessarily satisfy the other conditions in (4.12) also. To get a prepotential with such additional properties, we need to further change $\varphi^{o}$ into a new (further restricted) gauge $\varphi$, which preserves the property of being analytic, i.e. with $(1,0)$-potential components $A_{0}$ and $A_{-a}$ identically vanishing and with $A_{--}$satisfying the first pair of equations in (4.12). In order to determine this new analytic gauge $\varphi$, let us consider the $(2 n+2)$-dimensional involutive distribution $\widehat{\mathcal{D}} \subset T^{\mathbb{C}}\left(\mathcal{V} \times \mathcal{V} \times \mathrm{SL}_{2}(\mathbb{C}) \times G\right)$ generated by the holomorphic vector fields $H_{0}, H_{ \pm \pm}+A_{ \pm \pm}^{o}$ and $e_{-a}$. Then, for each point $\left(x, y, U_{o}, g\right)$ of the manifold $\widehat{S} \times G$, consider the unique integral leaf through $\left(x, y, U_{o}, g\right)$ of this distribution $\mathcal{T}^{\left(x, y, U_{o}, g\right)} \subset \mathcal{V} \times \mathcal{V} \times \mathrm{SL}_{2}(\mathbb{C}) \times G$. Now we may determine a $G$-equivariant $\mathfrak{g}$-valued holomorphic function $h_{--}$on $\mathcal{T}^{\left(x, y, U_{o}, g\right)}$ (the $G$-equivariance being with respect to the standard right action of $G$ on $\mathcal{V} \times \mathcal{V} \times \mathrm{SL}_{2}(\mathbb{C}) \times G$ and the adjoint $G$-action on $\left.\mathfrak{g}\right)$ satisfying the equations

$$
H_{0} \cdot h_{--}=-2 h_{--}, \quad H_{--} \cdot h_{--}=-H_{--} \cdot A_{--}^{o}-\left[h_{--}, A_{--}^{o}\right], \quad e_{-a} \cdot h_{--}=0 .
$$

Once again, the existence of such an $h$ can be checked using [16, Lemma 5.3]. Indeed, one can construct a solution to the first two conditions as follows. Along the image of $\mathcal{T}^{\left(x, y, U_{o}, g\right)}$ under the inverse gauge transformation $\widetilde{\varphi} \circ \varphi^{o-1}\left(=\varphi^{o-1}\right)$ (note that, by $G$ equivariance of the trivialisation, each intersection of the submanifold $\varphi^{o-1}\left(\mathcal{T}^{\left(x, y, U_{o}, g\right)}\right)$ with a vertical set $\{(\tilde{x}, \tilde{y})\} \times \mathrm{SL}_{2}(\mathbb{C}) \times G$ is entirely included in a submanifold of the form $\left.\{(\tilde{x}, \tilde{y})\} \times \mathrm{SL}_{2}(\mathbb{C}) \times\{g\}\right)$ we may consider a $G$-equivariant $\mathfrak{g}$-valued solution $\widetilde{h}_{--}$to the differential problem

$$
H_{0} \cdot \tilde{h}_{--}=-2 \tilde{h}_{--}, \quad H_{--} \cdot \tilde{h}_{--}=-H_{--} \cdot\left(A_{--}^{o} \circ \varphi^{o}\right), \quad e_{-a}^{o} \cdot \tilde{h}_{--}=0 .
$$

By [16, Lemma 5.3], applied to the pair of vector fields $H_{0}, H_{--}$in place of the pair $H_{0}, H_{++}$, such a solution $\widetilde{h}_{--}$exists. The corresponding function $h_{--}=\widetilde{h}_{--} \circ \varphi^{o-1}$ is therefore a solution to (4.13). Note that the solution $\widetilde{h}_{--}$to (4.14) is uniquely determined up to addition of a solution $\widetilde{k}_{--}$of the associated system

$$
H_{0} \cdot \widetilde{k}_{--}=-2 \widetilde{k}_{--}, \quad H_{--} \cdot \tilde{k}_{--}=0, \quad e_{-a}^{o} \cdot \tilde{k}_{--}=0
$$

and that such a solution $\widetilde{k}_{--}$can be assumed to take any desired valued at the points of $\mathcal{V} \times \mathcal{V} \times\left\{U_{o}\right\} \times G$. This can be checked by observing that an explicit expression for any holomorphic solution $\widetilde{k}_{--}$is actually determined in [16, Lemma 5.3] as

$$
\tilde{k}_{--}(x, y, U, g)=\sum_{\substack{m, n \geq 0 \\ m+n=2}} c_{m n}(x, y, g)\left(u_{-}^{1}\right)^{m}\left(u_{-}^{2}\right)^{n},
$$

with $U=\left(u_{ \pm}^{i}\right)$. Observing that the matrix $U_{o}=I_{2}$ has entries $u_{-}^{1}, u_{-}^{2}$ equal to 0,1 , respectively, by appropriately choosing the component $c_{02}(x, y, g)$, the function can take any desired value at the points of $\mathcal{V} \times \mathcal{V} \times\left\{U_{o}\right\} \times G$. Since $\varphi^{o}\left(\left(\mathcal{V} \times \mathcal{V} \times\left\{U_{o}\right\} \times G\right) \cap \widehat{S}\right) \subset$ $\left(\mathcal{V} \times \mathcal{V} \times\left\{U_{o}\right\} \times G\right) \cap \widehat{S}$, such a residual degree of freedom for the $\widetilde{h}_{--}$can be used to make the restriction $h_{--} \mid \widehat{S} \cap \mathcal{T}^{\left(x, U_{o}, g\right)}$ identically zero.

We combine the solutions along the leaves $\mathcal{T}^{\left(x, y, U_{o}, g\right)},\left(x, y, U_{o}, g\right) \in \widehat{S} \times G$, into a global solution of (4.13) on $\mathcal{V} \times \mathcal{V} \times \mathrm{SL}_{2}(\mathbb{C}) \times G$ and restrict such a globally defined $\mathfrak{g}$-valued map $h_{--}$to the submanifold $\mathcal{V} \times \mathcal{V} \times \mathrm{SL}_{2}(\mathbb{C}) \times\{e\} \simeq \mathcal{V} \times \mathcal{V} \times \mathrm{SL}_{2}(\mathbb{C})$. Then, along each integral leaf in $\mathcal{V} \times \mathcal{V} \times \mathrm{SL}_{2}(\mathbb{C})$ of the distribution spanned by $H_{\alpha}$ and $e_{-a}$, we may consider a new 
$\mathfrak{g}$-valued function $\psi^{\prime}$ satisfying the differential problem

$$
H_{0} \cdot \psi^{\prime}=0, \quad H_{--} \cdot \psi^{\prime}=h_{--}, \quad e_{-a} \cdot \psi^{\prime}=0 .
$$

The same argument as before shows the existence of such a solution. Moreover, the residual degree of freedom in the choice of the solution may be used to set it to 0 at each point of the form $\left(x, y, U_{o}\right)$. Combining the solutions along all the considered integral leaves, we get a global solution $\psi^{\prime}$ such that $\psi^{\prime}\left(x, y, U_{o}\right)=0$ for all $(x, y) \in \mathcal{V} \times \mathcal{V}$ and satisfying the differential problem

$$
H_{0} \cdot \psi^{\prime}=0, \quad H_{--} \cdot\left(H_{--} \cdot \psi^{\prime}\right)=-H_{--} \cdot A_{--}^{o}-\left[H_{--} \cdot \psi^{\prime}, A_{--}^{o}\right], \quad e_{-a} \cdot \psi^{\prime}=0 .
$$

Let us now consider the new gauge $\varphi$, obtained by applying to the analytic gauge $\varphi^{o}$ the gauge transformation $g_{(x, y)}=e^{\psi_{(x, y)}^{\prime}},(x, y) \in \mathcal{V} \times \mathcal{V}$. Recalling that $A_{0}^{o}=A_{-a}^{o}=0$, we see that the potential in the new gauge $\varphi$ has components $A_{0}, A_{--}, A_{-a}$ given by

$$
\begin{array}{ll}
A_{0}=e^{-\operatorname{ad}_{\psi^{\prime}}}\left(A_{0}^{o}+H_{0} \cdot \psi^{\prime}\right)=0, & A_{-a}=e^{-\mathrm{ad}_{\psi^{\prime}}}\left(A_{-a}^{o}+e_{-a} \cdot \psi^{\prime}\right)=0, \\
& A_{--}=e^{-\mathrm{ad}_{\psi^{\prime}}\left(A_{--}^{o}+H_{--} \cdot \psi^{\prime}\right) .}
\end{array}
$$

From this and (4.15), it follows that

$$
H_{--} \cdot A_{--}=e^{a d_{\psi^{\prime}}}\left(\left[H_{--} \cdot \psi^{\prime}, A_{--}^{o}\right]+H_{--} \cdot A_{--}^{o}+H_{--} \cdot\left(H_{--} \cdot \psi^{\prime}\right)\right)=0
$$

and, with a similar computation, that $H_{0} \cdot A_{--}=-2 A_{--}$and $e_{-a} \cdot A_{--}=0$. In other words, the new prepotential $A_{--}$satisfies all three conditions in the first line of (4.12). To check the last two normalising conditions, we recall at first that in any central frame, the corresponding potential $\widetilde{A}$ satisfies

$$
H_{0} \cdot \widetilde{A}_{-a}=-\widetilde{A}_{-a}, \quad H_{++} \cdot \widetilde{A}_{-a}=\widetilde{A}_{+a}, \quad H_{--} \cdot \widetilde{A}_{-a}=0 .
$$

Hence, if we differentiate the identity $\left.\psi^{o}\right|_{S}=0$ in directions tangent to $S$, using (4.11), (4.12) and commutation relations we get

$$
\left.e_{+a} \cdot \psi^{o}\right|_{S}=\left.H_{++} \cdot \psi^{o}\right|_{S}=\left.H_{--} \cdot \psi^{o}\right|_{S}+\left.\lambda^{a} \widetilde{A}_{-a}\right|_{S}=0 .
$$

Since $\left.e^{\psi^{o}}\right|_{S}=\mathrm{Id}$, we conclude that $\left.A_{--}^{o}\right|_{S}=\left.\widetilde{A}_{--}\right|_{S}+\left.H_{--} \cdot \psi^{o}\right|_{S}=-\left.\lambda^{a} \widetilde{A}_{-a}\right|_{S}$. Now, from (4.15), the property that $\widehat{S} \subset S$ and $\left.\psi^{\prime}\right|_{\widehat{S}}=\left.H_{--} \cdot \psi^{\prime}\right|_{\widehat{S}}=0$, the second line in (4.12) follows immediately.

The $\mathfrak{g}$-bridge $\psi$, the corresponding analytic gauge $\varphi$ and the associated prepotential $A_{--}$ established by Lemma 4.6 are called normalised at $\left(x_{o}, x_{o}, U_{o}\right)$.

Remark 4.7 The proof of the previous theorem shows that any prepotential $A_{--}$(not just the normalised ones) is such that $X_{-} \cdot A_{--}=0$ for any horizontal vector field in the distribution $\mathcal{D}_{-}$. On the other hand, we also have that:

(a) $F(V, \cdot)=0$ for any vertical vector field $V$ of $\mathcal{H}^{\mathbb{C}}(M)$, i.e. for any $V$ which is tangent to the vertical fibres $\{(x, y)\} \times \mathrm{SL}_{2}(\mathbb{C})$ of $\mathcal{H}^{\mathbb{C}}(M)$.

(b) The vertical anti-holomorphic distribution of $\mathcal{H}^{\mathbb{C}}(M)$ is spanned by right invariant vector fields along the fibres $\{x\} \times \mathrm{SL}_{2}(\mathbb{C})$, which therefore commute with the left invariant holomorphic vector field $H_{--}$.

(c) The holomorphic potential $A$ in an analytic gauge vanishes identically along the antiholomorphic vector fields of $\mathcal{H}^{\mathbb{C}}(M)$. This is due to Proposition 4.1 and the fact that the analytic gauges are holomorphic with respect to the complex structures of the extended $P^{\prime}$ over $\left(\mathcal{H}^{\mathbb{C}}(M), \mathbb{I}^{\mathbb{C}}\right)$. 
From (a), (b), (c) and the explicit expression of $F$ in terms of a potential, it follows that, for any anti-holomorphic vertical vector field $V^{01}$ of $\mathcal{H}^{\mathbb{C}}(M)$, we have $V^{01} \cdot A_{--}=0$. This and the above property $X_{-} \cdot A_{--}=0$ prove that for any prepotential $A_{--}$defined on some open set $\mathcal{W}$ of the complexified harmonic space $\left(\mathcal{H}^{\mathbb{C}}(M), \mathbb{I}^{\mathbb{C}}\right)$, the restriction $A_{--} \mid \mathcal{H}(M) \cap \mathcal{W}$ is also holomorphic with respect to the complex structure $\mathbb{I}$ of the harmonic space $\mathcal{H}(M)$.

\section{Existence, uniqueness and compactness theorems}

\subsection{Existence and uniqueness of an instanton with a given prepotential}

Theorem 5.1 Let $\mathcal{V} \subset M$ be open and simply connected. For any map $A_{--}: \mathcal{V} \times \mathrm{SL}_{2}(\mathbb{C}) \subset$ $\mathcal{H}(M) \rightarrow \mathfrak{g}$, which is holomorphic (i.e. with $X_{-} \cdot A_{--}=0$ for any $X_{-} \in \mathcal{D}_{-}$and holomorphic in the complex coordinates of $\left.\mathrm{SL}_{2}(\mathbb{C})\right)$ and satisfies

$$
H_{0} \cdot A_{--}=-2 A_{--},
$$

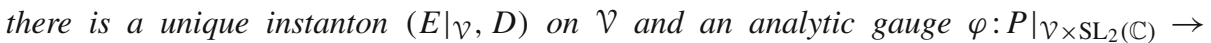
$\left(\mathcal{V} \times \mathrm{SL}_{2}(\mathbb{C})\right) \times G$, for which $A_{--}$is the prepotential in that gauge.

Proof In order to prove the existence of an associated instanton, we proceed as in the proof of [1, Thm. 4] and we consider an orbit $\{x\} \times \mathcal{O} \subset \mathcal{V} \times \mathrm{SL}_{2}(\mathbb{C})$ of the Borel subgroup $B \subset \mathrm{SL}_{2}(\mathbb{C})$, generated by $\left\langle H_{--}^{o}, H_{0}^{o}\right\rangle \subset \mathfrak{s l}_{2}(\mathbb{C})$, i.e.

$$
B=\left\{\left(\begin{array}{cc}
\zeta & 0 \\
z & \zeta^{-1}
\end{array}\right),(\zeta, z) \in \mathbb{C}^{*} \times \mathbb{C}\right\} \subset \mathrm{SL}_{2}(\mathbb{C})
$$

Along such an orbit, we may consider the unique $\mathfrak{g}$-valued connection for the $G$-bundle $\{x\} \times \mathcal{O} \times G$, whose $(1,0)$-potential is defined by $A\left(H_{--}\right)=A_{--}$and $A\left(H_{0}\right):=0$. Due to (5.1) and the fact that $\left[H_{0}, H_{--}\right]=-2 H_{--}$, such a connection has zero curvature. Hence, there exists a new holomorphic gauge $\varphi:\{x\} \times \mathcal{O} \times G \rightarrow\{x\} \times \mathcal{O} \times G$ which fixes all points of $\{x\} \times \mathcal{O} \times\{e\}$ and transforms $A$ into the identically vanishing potential. By (2.2), this is tantamount to saying that the associated gauge transformation $g_{(x, U)},(x, U) \in\{x\} \times \mathcal{O}$, is a solution to the differential problem

$$
H_{0} \cdot g=0, \quad H_{--} \cdot g+A_{--}=0,\left.\quad g\right|_{(x, U)}=e .
$$

Since the space of all $B$-orbits $\{x\} \times \mathcal{O}$ in $\mathcal{V} \times \mathrm{SL}_{2}(\mathbb{C})$ is diffeomorphic to $\mathcal{V} \times \mathbb{C} P^{1}$ and is therefore simply connected, all these new gauges combine into a globally defined gauge

$$
\varphi: \mathcal{V} \times \mathrm{SL}_{2}(\mathbb{C}) \times G \rightarrow \mathcal{V} \times \mathrm{SL}_{2}(\mathbb{C}) \times G,
$$

which maps each $B \times G$-orbit into itself and satisfies (5.2) at all points. We may now consider the $\mathfrak{g}$-valued map on $\mathcal{V} \times \mathrm{SL}_{2}(\mathbb{C})$

$$
A_{++}: \mathcal{V} \times \mathrm{SL}_{2}(\mathbb{C}) \rightarrow \mathfrak{g}, \quad A_{++}:=-\left(H_{++} \cdot g\right) g^{-1} .
$$

Combining this function with the $\mathfrak{g}$-valued maps $A_{--}$and $A_{0}=0$, we may construct the $G$-invariant vector fields $H_{0}^{h}, H_{ \pm \pm}^{h}$ on $\mathcal{V} \times \mathrm{SL}_{2}(\mathbb{C}) \times G$, which on the submanifold $V \times \mathrm{SL}_{2}(\mathbb{C}) \times\{e\}$ are

$$
\begin{aligned}
& H_{0}^{h}\left|\mathcal{v} \times \mathrm{SL}_{2}(\mathbb{C}) \times\{e\}:=H_{0}\right| \mathcal{v} \times \mathrm{SL}_{2}(\mathbb{C}) \times\{e\} \\
& H_{ \pm \pm}^{h}\left|\mathcal{v} \times \mathrm{SL}_{2}(\mathbb{C}) \times\{e\}:=H_{ \pm \pm}\right| \mathcal{v} \times \mathrm{SL}_{2}(\mathbb{C}) \times\{e\}+A_{ \pm \pm} .
\end{aligned}
$$


By real analyticity, there is an open $\mathcal{U} \subset \mathcal{H}^{\mathbb{C}}(M)$ with $\mathcal{U} \cap \mathcal{H}(M)=\mathcal{V} \times \mathrm{SL}_{2}(\mathbb{C})$, where these vector fields extend as holomorphic fields on $\mathcal{U} \times G$. Moreover, by the construction of $A_{--}$and of the map (5.3), along each fibre $\{x\} \times \mathrm{SL}_{2}(\mathbb{C}) \times G$, the functions $A_{0}:=0, A_{ \pm \pm}$ can be considered as the three components of the holomorphic potential of a connection for the $G$-bundle $\pi^{(x)}:\{x\} \times \mathrm{SL}_{2}(\mathbb{C}) \times G \rightarrow\{x\} \times \mathrm{SL}_{2}(\mathbb{C})$, which is transformed by the gauge $\varphi$ into the trivial potential. This means that the associated covariant derivative has identically vanishing curvature, i.e. $F\left(H_{\alpha}, H_{\beta}\right) \equiv 0$ for all $\alpha, \beta \in\{0,++,--\}$.

These connections on the submanifolds $\{x\} \times G$ of the open set $\mathcal{U} \times \mathrm{SL}_{2}(\mathbb{C}) \times G$ can be considered as restrictions of a $G$-connection on the (trivial) bundle $p: \mathcal{U} \times G \rightarrow \mathcal{U}$, with associated holomorphic potential $A: \mathcal{U} \rightarrow T^{*} \mathcal{U} \otimes \mathfrak{g}$ satisfying

$$
\begin{array}{ll}
A\left(H_{\alpha}\right):=A_{\alpha}, & \alpha \in\{0,++,--\}, \\
A\left(X_{-}\right):=0, & \text { for any } X_{-} \in \mathcal{D}_{-}, \\
A\left(e_{+a}\right):=-e_{-a} \cdot A_{++} & \text {for any frame field }\left(e_{+a}, e_{-b}\right) \text { generating } \mathcal{D}_{+} \oplus \mathcal{D}_{-} \\
& \text {and projecting onto the complex frames }\left(e_{ \pm a}\right) \text { of } M \\
& \text { described in Sect. 2.1 (for their existence, see below). }
\end{array}
$$

We now need to show that the curvature of the associated covariant derivative $D^{\prime}$ satisfies the following equalities for each $X_{ \pm}, Y_{ \pm} \in \mathcal{D}_{ \pm}$:

$$
\begin{aligned}
& F\left(X_{+}, Y_{+}\right)=0=F\left(X_{-}, Y_{-}\right), \\
& F\left(H_{\alpha}, X_{+}\right)=0=F\left(H_{\alpha}, X_{-}\right), \quad \alpha \in\{0,++,--\} .
\end{aligned}
$$

This would conclude the proof. Indeed, by Proposition 2.2 and Lemma 4.2, it would imply that the corresponding gauge field $\left(E^{\prime} \mid u, D^{\prime}\right)$ is the extension to $\mathcal{U} \subset \mathcal{H}^{\mathbb{C}}(M)$ of the lift of an instanton $\left(\left.E\right|_{\mathcal{V}}, D\right)$ on $\mathcal{V}$ and with $A_{--}$as prepotential. Further, by the above definition of the potential, the trivial gauge $\varphi=\operatorname{Id}_{\mathcal{V}} \times \mathcal{V} \times \mathrm{SL}_{2}(\mathbb{C}) \times G$ would be an analytic gauge for such an instanton. Hence, by Theorem 4.3, any instanton having prepotential $A_{--}$in such a gauge would necessarily coincide with $(E \mid \mathcal{v}, D)$.

In order to prove (5.6), for each given point $\left(x_{o}, y_{o}, U_{o}\right) \in \mathcal{U} \subset \mathcal{H}^{\mathbb{C}}(M)=M \times M \times$ $\mathrm{SL}_{2}(\mathbb{C})$ we select a (locally defined) collection of $\mathbb{I}^{(\mathbb{C})}$-holomorphic vector fields $\left(e_{+a}, e_{-a}\right)$, which generate the distribution $\mathcal{D}_{+} \oplus \mathcal{D}_{-}$and project to the complex vectors in $T^{\mathbb{C}} M$ determined by adapted frames of $M$ and described in Sect. 2.1. A collection of vector fields $e_{ \pm a}$ of this kind can be constructed through the following three step procedure:

(1) Pick a local section $\sigma: \mathfrak{U}^{\prime} \rightarrow O_{g}\left(\mathcal{V} \times \mathcal{V}, J_{\alpha}\right)$ of the bundle of adapted frames over $v \times v$.

(2) Consider the restrictions to the section $\sigma\left(\mathcal{U}^{\prime}\right)$ of the canonical horizontal vector fields $\nabla_{e_{ \pm a}}:=e_{ \pm a}+\Gamma_{ \pm a}^{J}$ determined by the Levi-Civita connection (here, the $\Gamma_{ \pm a}^{J}$ are the Christoffel symbols).

(3) Take the projections $\left(e_{ \pm a}\right)$ of the vector fields $\nabla_{e_{ \pm a}}:=e_{ \pm a}+\Gamma_{ \pm a}{ }_{I}^{J}$ onto the underlying open set $\mathfrak{U}^{\prime}$.

Since the Levi-Civita connection has vanishing torsion, the vector fields $e_{ \pm a}$ constructed in this way are such that

$$
\left[e_{+a}, e_{+b}\right]=0=\left[e_{-a}, e_{-a}\right] .
$$

Further, just by looking at the standard action of the $H_{\alpha}^{o} \in \mathfrak{s l}_{2}(\mathbb{C})$ on the elements $\left(h_{i}^{o} \otimes e_{a}^{o}\right) \subset$ $\mathbb{C}^{2} \otimes \mathbb{C}^{2 n} \simeq\left(\mathbb{H}^{n}\right)^{\mathbb{C}}$, the actions of the vector fields $H_{\alpha}$ of $\mathcal{H}^{\mathbb{C}}(M)=M \times M \times \mathrm{SL}_{2}(\mathbb{C})$ on the $e_{ \pm a}$ are

$$
\left[H_{0}, e_{ \pm a}\right]= \pm e_{ \pm a}, \quad\left[H_{ \pm \pm}, e_{ \pm a}\right]=0, \quad\left[H_{ \pm \pm}, e_{\mp a}\right]=e_{ \pm a}
$$


Hence, by (2.5) and the assumption $A\left(H_{0}\right)=A\left(e_{-a}\right)=0$,

$$
F\left(H_{0}, e_{-a}\right)=F\left(e_{-a}, e_{-b}\right)=0 .
$$

From condition $A\left(e_{+a}\right)=-e_{-a} \cdot A\left(H_{++}\right)$, we also have

$$
\begin{aligned}
& F\left(H_{++}, e_{-a}\right)=-e_{-a} \cdot A\left(H_{++}\right)-A\left(e_{+a}\right)=0, \\
& F\left(H_{--}, e_{-a}\right)=-e_{-a} \cdot A\left(H_{--}\right)=-e_{-a} \cdot A_{--} \stackrel{(5.1)}{=} 0 .
\end{aligned}
$$

Finally, from (5.9), (5.10), the property $F\left(H_{\alpha}, H_{\beta}\right)=0$ and Bianchi identities

$$
\sum_{\substack{\text { cyclic permutations } \\ \text { of }(1,2,3)}} D_{X_{i_{1}}}^{\prime} F\left(X_{i_{2}}, X_{i_{3}}\right)+F\left(X_{i_{1}},\left[X_{i_{2}}, X_{i_{3}}\right]\right)=0
$$

with $X_{1}, X_{2}, X_{3}$ equal to the triple $H_{++}, H_{--}, e_{-a}$ or to the triple $H_{++}, H_{0}, e_{-a}$, we get that $F\left(H_{--}, e_{+b}\right)=0=F\left(H_{0}, e_{+b}\right)$. All this shows that

$$
\begin{aligned}
F\left(e_{-a}, e_{-b}\right) & =0, \\
F\left(H_{\alpha}, e_{-a}\right) & =0, \quad \alpha \in\{0,++,--\}, \\
F\left(H_{0}, e_{+a}\right) & =F\left(H_{--}, e_{+a}\right)=0 .
\end{aligned}
$$

Now, in order to conclude the proof, we need the following

Lemma 5.2 The components $F\left(H_{++}, e_{+a}\right), F\left(e_{+a}, e_{+b}\right), 1 \leq a, b \leq 2 n$, of the abovedefined covariant derivative $D^{\prime}$ are identically vanishing.

Proof of Lemma. We recall that, by real analyticity, $F\left(H_{++}, e_{+a}\right), F\left(e_{+a}, e_{+b}\right)$ are extended as holomorphic functions on an $\mathrm{SL}_{2}(\mathbb{C})$-invariant neighbourhood $\mathcal{U} \times\{e\} \subset \mathcal{H}^{\mathbb{C}}(M) \times\{e\}$ of $\mathcal{V} \times \mathrm{SL}_{2}(\mathbb{C}) \times\{e\}$. Note also that, due to (5.12) and Bianchi identities among the vector fields $H_{0}, H_{++}, e_{+a}$ or $H_{++}, H_{--}, e_{+a}$, respectively, we have that

$$
\begin{aligned}
& D_{H_{0}}^{\prime} F\left(H_{++}, e_{+a}\right)=3 F\left(H_{++}, e_{+a}\right), \\
& D_{H_{--}}^{\prime} F\left(H_{++}, e_{+a}\right)=0 .
\end{aligned}
$$

If we consider the (holomorphic extensions of the) $G$-invariant vector fields $H_{0}^{h}, H_{ \pm \pm}^{h}$ on $\mathcal{V} \times \mathrm{SL}_{2}(\mathbb{C}) \times G$ defined by (5.4) and the $G$-equivariant function

$$
\mathcal{F}_{+++a}: \mathcal{U} \times G \rightarrow \mathfrak{g},\left.\quad \mathcal{F}_{+++a}\right|_{\mathfrak{U} \times\{e\}}:=F\left(H_{++}, e_{+a}\right),
$$

we see that (5.13) is equivalent to the system of equations for the $\mathcal{F}_{+++a}$

$$
\begin{aligned}
& H_{0}^{h} \cdot \mathcal{F}_{+++a}=3 \mathcal{F}_{+++a}, \\
& H_{--}^{h} \cdot \mathcal{F}_{+++a}=0 .
\end{aligned}
$$

By [16, Lemma 5.3] (or, more precisely, by its analogue involving the vector field $H_{--}$ in place of $H_{++}$) the restriction of (5.14) to each orbit $\mathcal{O} \subset \mathcal{U} \times G$ of the $\mathrm{SL}_{2}(\mathbb{C})$-action generated by the $H_{\alpha}^{h}$, admits exactly one solution, namely the identically zero function. It follows that $\mathcal{F}_{+++a}=F\left(H_{++}, e_{+a}\right) \equiv 0$ on $\mathcal{V} \times \mathrm{SL}_{2}(\mathbb{C}) \times G$.

Let us now focus on the components $F\left(e_{+a}, e_{+b}\right)$. By (5.12) and Bianchi identities (5.11) among the vector fields $H_{0}, e_{+a}, e_{+b}$ and $H_{--}, e_{+a}, e_{+b}$, we have that

$$
\begin{aligned}
& D_{H_{0}}^{\prime} F\left(e_{+a}, e_{+b}\right)=2 F\left(H_{++}, e_{+a}\right), \\
& D_{H_{--}}^{\prime} F\left(e_{+a}, e_{+b}\right)=F\left(e_{+a}, e_{-b}\right)-F\left(e_{+b}, e_{-a}\right)=0,
\end{aligned}
$$


where the last equality is a consequence of the fact that $A_{-a}=0$ and of

$$
F\left(e_{+a}, e_{-b}\right)=e_{-b} \cdot\left(e_{-a} \cdot A_{++}\right) \stackrel{\left[e_{-a}, e_{-b}\right]=0}{=} e_{-a} \cdot\left(e_{-b} \cdot A_{++}\right)=F\left(e_{+b}, e_{-a}\right) .
$$

By the same argument as before, the unique $G$-equivariant extension of the $\mathfrak{g}$-valued function $\mathcal{F}_{++a b}:=F\left(e_{+a}, e_{+b}\right)$ is solution to the differential problem $H_{0}^{h} \cdot \mathcal{F}_{++a b}=2 \mathcal{F}_{++a b}$ and $H_{--}^{h} \cdot \mathcal{F}_{++a b}=0$. As above, by [16, Lemma 5.3], we get that $\mathcal{F}_{++a b}=F\left(e_{+a}, e_{+b}\right)$ vanishes identically.

From (5.12) and Lemma 5.2, all conditions in (5.6) are satisfied.

\subsection{Bounds for normalised prepotentials}

Let $\mathcal{V} \times \mathrm{SL}_{2}(\mathbb{C})$ be an $\mathrm{SL}_{2}(\mathbb{C})$-invariant open subset of $\mathcal{H}(M)$ with $\mathcal{V} \subset M$ relatively compact and simply connected and such that $\mathcal{V} \times \mathrm{SL}_{2}(\mathbb{C})$ is a domain for both an expcentral gauge $\tilde{\varphi}$ and an analytic gauge $\varphi$ for an instanton $(E, D)$ which is normalised around $\left(x_{o}, x_{o}, U_{o}:=I_{2}\right) \in \mathcal{V} \times \mathcal{V} \times \mathrm{SL}_{2}(\mathbb{C})$, with $x_{o} \in \mathcal{V}$. Let us also denote by $A_{--}: \mathcal{V} \times \mathrm{SL}_{2}(\mathbb{C}) \rightarrow \mathfrak{g}$ the corresponding normalised prepotential.

Theorem 5.3 There exists a relatively compact simply connected neighbourhood $\mathcal{V}^{\prime} \subset \mathcal{V}$ of $x_{o}$, such that for each compact subset $K \subset \overline{V^{\prime}} \times \mathrm{SL}_{2}(\mathbb{C})$, there is a constant $c_{K}, \overline{\mathcal{V}}>0$, depending just on the compact sets $K$ and $\overline{\mathcal{V}}$, such that

$$
\left\|A_{--}\right\|_{\mathcal{C}^{0}(K, \mathfrak{g})} \leq c_{K, \overline{\mathcal{V}}}\|F\|_{\mathcal{C}^{0}(\overline{\mathcal{V}}, \mathfrak{g})}^{(\widetilde{\widetilde{c}})} .
$$

Proof Let $\widehat{S} \subset \mathcal{V} \times \mathcal{V} \times \mathrm{SL}_{2}(\mathbb{C})$ be the complex submanifold passing through $\left(x_{o}, x_{o}, I_{2}\right)$ and defined in $\$ 4.4$, and for each $(x, y, U) \in \widehat{S}$, let $\mathcal{S}^{(x, y, U)}$ be the unique integral leaf through $(x, y, U)$ of the complex distribution generated by the holomorphic vector fields $e_{-a}$ and $H_{0}$ of $\mathcal{H}^{\mathbb{C}}(M)$. Let also $\mathcal{V}^{\prime}$ be a relatively compact connected open subset of $\mathcal{V}$ which contains $\left(x_{o}, x_{o}, I_{2}\right)$ and such that the $\mathrm{SL}_{2}(\mathbb{C})$-invariant set $\overline{\mathcal{V}^{\prime}} \times \overline{\mathcal{V}^{\prime}} \times \mathrm{SL}_{2}(\mathbb{C})$ is included in

$$
\overline{V^{\prime}} \times \overline{V^{\prime}} \times \mathrm{SL}_{2}(\mathbb{C}) \subset \bigcup_{(x, y, U) \in \widehat{S}} \mathcal{S}^{(x, y, U)} \cap\left(\mathcal{V} \times \mathcal{V} \times \mathrm{SL}_{2}(\mathbb{C})\right) .
$$

The existence of such a $\mathcal{V}^{\prime}$ is guaranteed by the fact that the family of integral leaves $\mathcal{S}^{(x, y, U)}$ is $\mathrm{SL}_{2}(\mathbb{C})$-invariant. The condition (5.18) is chosen to ensure that any point of $\overline{V^{\prime}} \times \overline{V^{\prime}} \times$ $\mathrm{SL}_{2}(\mathbb{C}) \subset \mathcal{H}^{\mathbb{C}}(M)$ lies in some (connected) intersection $\mathcal{S}^{(x, y, U)} \cap\left(\mathcal{V} \times \mathcal{V} \times \mathrm{SL}_{2}(\mathbb{C})\right)$.

Let $K \subset \overline{V^{\prime}} \times \mathrm{SL}_{2}(\mathbb{C}) \subset \mathcal{H}(M)\left(\subset \mathcal{H}^{\mathbb{C}}(M)\right)$ be compact and denote by $K^{\prime} \subset \widehat{S}$ the set of points $(x, y, U) \in \widehat{S}$ such that $\mathcal{S}^{(x, y, U)} \cap K \neq \emptyset$. The set $K^{\prime}$ is compact. Indeed, it is the intersection between $\widehat{S}$ and the compact set of the (regular) orbits of the points of $K$ by the action on $\mathcal{V} \times \mathcal{V} \times \mathrm{SL}_{2}(\mathbb{C})$ of the local group generated by the holomorphic vector fields $H_{0}$ and $e_{-a}$.

Since $A_{--}$satisfies (4.12), by integration of the conditions $e_{-a} \cdot A_{--}=0$ and $H_{0} \cdot A_{--}=$ $-2 A_{--}$along each connected intersection $\mathcal{S}^{(x, y, U)} \cap \mathcal{V} \times \mathcal{V} \times \mathrm{SL}_{2}(\mathbb{C})$, it follows that there exists a constant $C_{\overline{\mathcal{V}}}>0$, depending only on $\overline{\mathcal{V}}$, such that

$$
\left\|A_{--}\right\|_{\mathcal{C}^{0}(K, \mathfrak{g})} \leq C_{\overline{\mathcal{V}}}\left(\sup _{(x, y, U) \in K^{\prime} \subset \widehat{S}}\left\|A_{--}\right\|\right)=C_{\overline{\mathcal{V}}}\left\|A_{--}\right\|_{\mathcal{C}^{0}\left(K^{\prime}, \mathfrak{g}\right)} .
$$

On the other hand, by the second line in (4.12),

$$
\left\|A_{--}\right\|_{\mathcal{C}^{0}\left(K^{\prime}, \mathfrak{g}\right)} \leq C_{K^{\prime}}^{\prime}\|\widetilde{A}\|_{\mathcal{C}^{0}\left(K^{\prime}, \mathfrak{g}\right)}
$$


for some constant $C_{K^{\prime}}^{\prime}$ depending only on the set $K^{\prime}$ or, equivalently, only on the compact set $K$. From this and the fact that $\widetilde{A}$ does not depend on the coordinates of $\mathrm{SL}_{2}(\mathbb{C})$, we infer that $\left\|A_{--}\right\|_{\mathcal{C}^{0}(K, \mathfrak{g})} \leq C_{\bar{v}} C_{K^{\prime}}^{\prime}\|\widetilde{A}\|_{\mathcal{C}^{0}\left(p_{1}\left(K^{\prime}\right), \mathfrak{g}\right)}$, where $p_{1}: \mathcal{H}(M)=M \times \mathrm{SL}_{2}(\mathbb{C}) \rightarrow M$ is the projection onto the first factor. Since $p_{1}\left(K^{\prime}\right) \subset \overline{\mathcal{V}}$, the claim follows from (4.3).

\subsection{The second prepotential and the curvature of instantons}

Let $\mathcal{V} \times \mathrm{SL}_{2}(\mathbb{C})$ be an $\mathrm{SL}_{2}(\mathbb{C})$-invariant open subset of $\mathcal{H}(M)$ with simply connected $\mathcal{V} \subset M$, which is domain for both an exp-central gauge $\widetilde{\varphi}$ and a (not necessarily normalised) analytic gauge $\varphi$. Further, let $A_{--}: \mathcal{V} \times \mathrm{SL}_{2}(\mathbb{C}) \rightarrow \mathfrak{g}$ be the prepotential of an instanton $\left(E^{\prime}, D^{\prime}\right)$ in the analytic gauge $\varphi$.

Definition 5.4 The second prepotential in the analytic gauge $\varphi$ is the $\mathfrak{g}$-valued map $A_{++}:=$ $A\left(H_{++}\right): \mathcal{V} \times \mathrm{SL}_{2}(\mathbb{C}) \rightarrow \mathfrak{g}$, determined by the evaluation of the holomorphic $(1,0)$-potential $A$ of the extension on $\mathcal{H}^{\mathbb{C}}(M)$ of the pair $\left(P^{\prime}, \omega^{\prime}\right)$ along the vector field $H_{++}$.

In what follows, to keep a clear distinction between $A_{--}$and $A_{++}$, we sometimes call $A_{--}$the first prepotential. Either function yields a complete local description of instantons on hk manifolds, since either one is completely determined by the other. However, in our framework, some features of the two descriptions are complementary:

(1) $A_{--}$is holomorphic on $(\mathcal{H}(M), \mathbb{I})$ and is a solution to the simple first-order linear equation $H_{0} \cdot A_{--}=-2 A_{--}$. If no normalisation is taken, there are no further restrictions. However, there is no direct way to compute the curvature tensor from $A_{--}$.

(2) $A_{++}$satisfies a (set of) second-order nonlinear equations, but in terms of it the curvature is given by the simple formula,

$$
\mathcal{F}^{\varphi}\left(e_{+a}, e_{-b}\right)=e_{-a} \cdot\left(e_{-b} \cdot A_{++}\right),
$$

i.e. the non-trivial components of the curvature are just the second-order derivatives of $A_{++}$along the anti-holomorphic directions $e_{-a}$.

The above-mentioned nonlinear equation for $A_{++}$has been used in various contexts in the physics literature, where it is known as the Leznov equation (see, for example, [12$15,27,35]$ ). The next lemma provides useful relations between the $\mathcal{C}^{k}$-norms of the two types of prepotentials.

Proposition 5.5 Let $A_{--}: \mathcal{V} \times \mathrm{SL}_{2}(\mathbb{C}) \rightarrow \mathfrak{g}$ be the (first) prepotential for an instanton and $A_{++}$the corresponding second prepotential. Then:

(1) $A_{--}$is the unique solution to the differential problem for the unknown $B_{--}$,

$$
H_{++} \cdot B_{--}=H_{--} \cdot A_{++}-\left[A_{++}, B_{--}\right], \quad H_{0} \cdot B_{--}=-2 B_{--} .
$$

A similar claim holds for $A_{++}$, provided appropriate sign changes are made.

(2) For each $k \geq 1$, there exist constants $M_{k}, M_{k}^{\prime}>0$, depending only on $k$, such that for $x \in \mathcal{V}$,

$$
\begin{aligned}
\left\|\left.A_{--}\right|_{\{x\} \times \mathrm{SU}_{2}}\right\|_{\mathcal{C}^{k}\left(\mathrm{SU}_{2}, \mathfrak{g}\right)} & \leq M_{k}\left\|\left.A_{++}\right|_{\{x\} \times \mathrm{SU}_{2}}\right\|_{\mathcal{C}^{k}\left(\mathrm{SU}_{2}, \mathfrak{g}\right)}, \\
\left\|\left.A_{++}\right|_{\{x\} \times \mathrm{SU}_{2}}\right\|_{\mathcal{C}^{k}\left(\mathrm{SU}_{2}, \mathfrak{g}\right)} & \leq M_{k}^{\prime}\left\|\left.A_{--}\right|_{\{x\} \times \mathrm{SU}_{2}}\right\|_{\mathcal{C}^{k}\left(\mathrm{SU}_{2}, \mathfrak{g}\right)} .
\end{aligned}
$$


Proof (1) Let $\left(\left.E^{\prime}\right|_{\mathcal{V} \times \mathrm{SL}_{2}(\mathbb{C})}, D^{\prime}\right)$ be the lifted instanton, for which $A_{ \pm \pm}, A_{0}(=0)$ are components of the potential in some analytic gauge $\varphi$. The expression in terms of curvature components of the identity $\mathcal{F}^{\varphi}\left(H_{++}, H_{--}\right)=0$ shows that $A_{--}$solves (5.20). This solution is unique. Indeed, if there were another solution to (5.20), say $A_{--}^{\prime}$, the $\mathfrak{g}$ valued map $\widetilde{B}_{--}:=\operatorname{Ad}_{\Psi}\left(A_{--}^{\prime}-A_{--}\right)$(here $\Psi$ is the gauge transformation from $\varphi$ to an exp-central gauge) would be a solution to the differential problem $H_{++} \cdot \widetilde{B}_{--}=0$, $H_{0} \cdot \widetilde{B}_{--}=-2 \widetilde{B}_{--}$. This fact and [16, Lemma 5.3] would imply that $\widetilde{B}_{--}=0$. Interchanging the signs, the corresponding claim for $A_{++}$follows.

(2) Consider the real basis for $\mathfrak{s u}_{2} \subset \mathfrak{s l}_{2}(\mathbb{C})$ given by the elements

$$
\begin{aligned}
G_{1}^{o} & :=H_{++}^{o}-H_{--}^{o}=\left(\begin{array}{cc}
0 & 1 \\
-1 & 0
\end{array}\right), \quad G_{2}^{o}:=i H_{++}^{o}+i H_{--}^{o}:=\left(\begin{array}{ll}
0 & i \\
i & 0
\end{array}\right), \\
G_{0}^{o} & :=i H_{0}^{o}=\left(\begin{array}{cc}
i & 0 \\
0 & -i
\end{array}\right),
\end{aligned}
$$

and let $G_{0}=i H_{0}, G_{1}=H_{++}-H_{--}, G_{2}=i H_{++}+i H_{--}$be the corresponding vector fields on $\mathcal{H}(M)=M \times \mathrm{SL}_{2}(\mathbb{C})$. Note that, for each $(x, U) \in M \times \mathrm{SU}_{2}$, the real vectors $\left.G_{\alpha}\right|_{(x, U)}, 1 \leq \alpha \leq 3$, give a frame for the tangent space of the totally real submanifold $\{x\} \times \mathrm{SU}_{2}$ of $\{x\} \times \mathrm{SL}_{2}(\mathbb{C})$.

Let $\left(E^{\prime} \mid \mathcal{V} \times \mathrm{SL}_{2}(\mathbb{C}), D^{\prime}\right)$ be the lifted instanton, for which $A_{ \pm \pm}, A_{0}$ are components of the potential in some analytic gauge $\varphi$. Since $A_{0}=0$ and the curvature $F^{\prime}$ of $\left(E^{\prime} \mid \mathcal{V} \times \mathrm{SL}_{2}(\mathbb{C}), D^{\prime}\right)$ vanishes identically along any vector field that is tangent to the fibres of $\mathcal{H}(M)$, at each $(x, U) \in\{x\} \times \mathrm{SU}_{2}$ we have that

$$
\begin{aligned}
0 & =i F^{\prime}\left(H_{0}, H_{--}\right)=i H_{0} \cdot A_{--}+2 i A_{--}=G_{0} \cdot A_{--}+2 i A_{--}, \\
0 & =2 F^{\prime}\left(H_{++}, H_{--}\right)=2 H_{++} \cdot A_{--}-2 H_{--} \cdot A_{++}+2\left[A_{++}, A_{--}\right]= \\
& =\left(G_{1}-i G_{2}\right) \cdot A_{--}+\left(G_{1}+i G_{2}\right) \cdot A_{++}+2\left[A_{++}, A_{--}\right] .
\end{aligned}
$$

Hence, the restrictions $V_{--}:=\left.A_{--}\right|_{\{x\} \times \mathrm{SU}_{2}}, W_{++}:=\left.A_{++}\right|_{\{x\} \times \mathrm{SU}_{2}}$ solve the system

$$
\begin{aligned}
& G_{0} \cdot V_{--}+2 i V_{--}=0, \\
& \left(G_{1}-i G_{2}\right) \cdot V_{--}+2 \operatorname{ad}_{W_{++}}\left(V_{--}\right)=-\left(G_{1}+i G_{2}\right) \cdot W_{++} .
\end{aligned}
$$

We claim that if (5.25) is considered as a system of equations for $V_{--}$with coefficients determined by $W_{++}$, then it is equivalent to a system given by an appropriate first-order elliptic operator $\mathcal{P}$ with trivial kernel (for definition and first properties of elliptic operators, we refer to [7, Appendix G]). To check this, consider the subbundle $\mathcal{E}$ of the bundle $\mathfrak{P}:=$ $\mathrm{SU}_{2} \times(\mathfrak{g} \times \mathfrak{g} \times \mathfrak{g} \times \mathfrak{g}) \rightarrow \mathrm{SU}_{2}$, defined by

$$
\mathcal{E}=\{(x, U ; X, Y, Z, W) \in \mathfrak{P}: \quad X=Z, \quad Y=W\} .
$$

We consider $\mathcal{E}$ equipped with the Hermitian product along the fibres determined by (2.8). Then, define

$$
\begin{aligned}
\mathcal{P} & : \mathcal{C}^{\infty}\left(M \times \mathrm{SU}_{2}, \mathcal{E}\right) \underset{\mathcal{C}^{\infty}\left(M \times \mathrm{SU}_{2}, \mathcal{E}\right),}{\longrightarrow} \\
& \mathcal{P}\left(\begin{array}{c}
X \\
Y \\
X \\
Y
\end{array}\right):=\left(\begin{array}{cccc}
G_{0}+2 i I & G_{1}+i G_{2}+2 \mathrm{ad}_{\overline{W_{++}}} & 0 & 0 \\
G_{1}-i G_{2}+2 \mathrm{ad}_{W_{++}} & G_{0}-2 i I & 0 & 0 \\
0 & 0 & -G_{0}-2 i I & G_{1}+i G_{2}+2 \mathrm{ad}_{\overline{W_{++}}} \\
0 & 0 & G_{1}-i G_{2}+2 & -G_{0}+2 i I
\end{array}\right) \cdot\left(\begin{array}{c}
X \\
Y \\
X \\
Y
\end{array}\right)
\end{aligned}
$$


One can directly check that $\mathcal{P}$ is a first-order elliptic operator. Moreover, $\operatorname{ker} \mathcal{P}=\{0\}$. Indeed, a quadruple $(X, Y, X, Y)$ is in ker $\mathcal{P}$ if and only if $X$ and $\bar{Y}$ are both solutions on $\mathrm{SU}_{2}$ to the system of equations for $\mathfrak{g}$-valued maps $f$

$$
\begin{aligned}
& H_{0} \cdot f=-2 f, \\
& H_{++} \cdot f+\operatorname{ad}_{W_{++}}(f)=0 .
\end{aligned}
$$

By applying the inverse of a gauge transformation from a central to the analytic gauge $\varphi$, the (flat) connection on $\mathrm{SU}_{2}$, determined by the potential $\left(A_{0}=0, A_{++}, A_{---}\right)$, is transformed into the (flat) connection determined by a potential with trivial components $\left(A_{0}^{\prime}=0, A_{++}^{\prime}=0, A_{--}^{\prime}=0\right)$. In particular, the solution $f$ to (5.27) is transformed into a solution $\widetilde{f}$ of the system

$$
\begin{aligned}
& H_{0} \cdot \tilde{f}=-2 \tilde{f}, \\
& H_{++} \cdot \tilde{f}=0 .
\end{aligned}
$$

By [16, Lemma 5.3] (applied to each component of the matrix valued function $\tilde{f}$ ), we get that $\tilde{f}$ is equal to 0 . This shows that $(5.28)$ has $(X, Y, X, Y)=(0,0,0,0)$ as the unique solution.

We may now conclude the proof of (2). In fact, it suffices to observe that (5.25) is equivalent to saying that the section of $\mathcal{E}$, given by the quadruple $\left(V_{--}, \overline{V_{--}}, V_{--}, \overline{V_{--}}\right)$, is a solution to the differential problem

$$
\mathcal{P}\left(\begin{array}{l}
V_{--} \\
\overline{V_{--}} \\
\overline{V_{--}}
\end{array}\right)=\left(\begin{array}{l}
-\left(G_{1}-i G_{2}\right) \cdot \overline{W_{++}} \\
-\left(G_{1}+i G_{2}\right) \cdot W_{++} \\
-\left(G_{1}-i G_{2}\right) \cdot \overline{W_{++}} \\
-\left(G_{1}+i G_{2}\right) \cdot W_{++}
\end{array}\right)
$$

Since $\mathcal{P}$ is elliptic with ker $\mathcal{P}=\{0\}$, classical Schauder estimates (see, for example, [7, Appendix H]) imply that, for each $k \geq 1$ there are constants $N_{k}, M_{k}>0$ such that

$$
\left\|V_{--}\right\|_{\mathcal{C}^{k}\left(\mathrm{SU}_{2}, \mathfrak{g}\right)} \leq N_{k}\left\|\left(\begin{array}{l}
-\left(G_{1}-i G_{2}\right) \cdot \overline{W_{++}} \\
-\left(G_{1}+i G_{2}\right) \cdot W_{++} \\
-\left(G_{1}-i G_{2}\right) \cdot W_{++} \\
-\left(G_{1}+i G_{2}\right) \cdot W_{++}
\end{array}\right)\right\|_{\mathcal{C}^{k-1}\left(\mathrm{SU}_{2}, \mathfrak{g}\right)} \leq M_{k}\left\|W_{++}\right\|_{\mathcal{C}^{k}\left(\mathrm{SU}_{2}, \mathfrak{g}\right)} .
$$

This gives (5.21). The proof of (5.22) is similar.

\subsection{The local compactness theorem}

To conclude this paper, as an example of the utility of the harmonic space formulation, we present a streamlined proof of Uhlenbeck, Nakajima and Tian's celebrated local compactness theorem for Yang-Mills fields in the specific case of hk instantons.

From the classical estimates in [30,37] (see also [36,43]), we know that for any geodesic ball $B_{R}=B_{R}\left(x_{o}\right)$ of radius $R$ of an $m$-dimensional Riemannian manifold $(M, g)$, the $\mathcal{C}^{0}$-norms of curvatures of Yang-Mills fields are controlled by their corresponding $L^{2}$ - or $L^{\frac{m}{2}}$-norms and $R$. In fact, there are constants $\varepsilon, C, c_{m}>0$ such that

$$
\begin{array}{llll}
\|F\|_{L^{2}\left(B_{R}\right)}<\varepsilon R^{m-4} & \text { implies } & \|F\|_{\mathcal{C}^{0}\left(B_{R / 4}, \mathfrak{g}\right)} & \leq \frac{C}{R^{\frac{m}{2}}}\|F\|_{L^{2}\left(B_{R}\right)} \\
\|F\|_{L^{\frac{m}{2}\left(B_{R}\right)}}<c_{m} & \text { implies } & \|F\|_{\mathcal{C}^{0}\left(B_{\frac{R}{2}}, \mathfrak{g}\right)} \leq \frac{2^{m} C}{R^{n}}\|F\|_{L^{2}\left(B_{\frac{R}{2}}\right)}
\end{array}
$$

Combining these estimates with Theorem 5.3 yields 
Theorem 5.6 (Local compactness theorem for instantons on hk manifolds) Let $B_{R}=$ $B_{R}\left(x_{o}\right) \subset M$ be a geodesic ball in a 4 -dimensional $h k$ manifold $\left(M, g, J_{\alpha}\right)$, which is included in a relatively compact neighbourhood $V^{\prime}$ of $x_{o}$ where Theorem 5.3 holds. Further let $\left(\left.E\right|_{B_{R}}=B_{R} \times V, D^{(k)}\right)$ be a sequence of (trivialised) instantons, each with the same compact structure group $G^{o}$ and corresponding normalised prepotential $A_{--}^{(k)}$ on $B_{R} \times \mathrm{SL}_{2}(\mathbb{C})$.

If the curvatures are such that $\left\|F^{(k)}\right\|_{L^{2}\left(B_{R}\right)}<\varepsilon R^{4(n-1)}$ for all $k$, with $\varepsilon>0$ as in (5.30), then there exists a subsequence $\left(\left.E\right|_{B_{R}}, D^{\left(k_{n}\right)}\right)$, whose curvatures $F^{\left(k_{n}\right)}$ converge uniformly to the curvature of a limit instanton $\left(\left.E\right|_{B_{R}}, D^{(\infty)}\right)$. The same conclusion holds if the curvatures are such that $\left\|F^{(k)}\right\|_{L^{2 n}\left(B_{2 R}\right)}<c_{4 n}$ with constant $c_{4 n}>0$ as in (5.31).

Proof By (5.30), (5.31), (5.17), the sequence of normalised holomorphic prepotentials $A_{--}^{(k)}$ is uniformly bounded on any compact subset $K$ of $B_{R} \times \mathrm{SL}_{2}(\mathbb{C})$. It follows from Montel's theorem that there is a subsequence $A_{--}^{\left(k_{n}\right)}$ converging uniformly on compacta to a holomorphic map $A_{--}^{(\infty)}$, which is the prepotential of some instanton due to Theorem 5.1. Using (5.22), we may also assume that the second prepotentials $A_{++}^{\left(k_{n}\right)}$ and all their derivatives converge uniformly on compacta to the second prepotential $A_{++}^{(\infty)}$ and its derivatives corresponding to the instanton determined by $A_{--}^{(\infty)}$. Thus, by (5.19), the curvatures converge uniformly on compacta as well.

Acknowledgements Open access funding provided by Max Planck Society. One of us (CD) thanks Hermann Nicolai and the Albert Einstein Institute for providing an excellent research environment.

Open Access This article is distributed under the terms of the Creative Commons Attribution 4.0 International License (http://creativecommons.org/licenses/by/4.0/), which permits unrestricted use, distribution, and reproduction in any medium, provided you give appropriate credit to the original author(s) and the source, provide a link to the Creative Commons license, and indicate if changes were made.

\section{References}

1. Alekseevsky, D.V., Cortes, V., Devchand, C.: Yang-Mills connections over manifolds with Grassmann structure. J. Math. Phys. 44, 6047-6074 (2003)

2. Atiyah, M.F.: Geometry of Yang-Mills Fields. Scuola Normale Superiore Pisa, Pisa (1979)

3. Atiyah, M.F., Hitchin, N.J., Drinfel'd, V.G., Manin, Yu.I.: Construction of instantons. Phys. Lett. A 65 , 185-187 (1978)

4. Atiyah, M.F., Hitchin, N.J., Singer, I.M.: Self-duality in four-dimensional Riemannian geometry. Proc. R. Soc. Lond. Ser. A 362, 425-461 (1978)

5. Atiyah, M.F., Ward, R.S.: Instantons and algebraic geometry. Commun. Math. Phys. 55, 117-124 (1977)

6. Belavin, A.A., Polyakov, A.M., Schwartz, A.S., Tyupkin, Y.S.: Pseudoparticle solutions of the Yang-Mills equations. Phys. Lett. B 59, 85-87 (1975)

7. Besse, A.: Einstein Manifolds. Springer, Berlin (1987)

8. Bourguignon, J.P., Lawson, H.B., Simons, J.: Stability and gap phenomena for Yang-Mills fields. Proc. Nat. Acad. Sci. USA 76, 1550-1553 (1979)

9. Corrigan, E., Devchand, C., Fairlie, D.B., Nuyts, J.: First-order equations for gauge fields in spaces of dimension greater than four. Nucl. Phys. B 214, 452-464 (1983)

10. Corrigan, E., Fairlie, D.B., Goddard, P., Yates, R.G.: The construction of self-dual solutions to SU(2) gauge theory. Commun. Math. Phys. 58, 223-240 (1978)

11. Corrigan, E., Goddard, P., Kent, A.: Some comments on the ADHM construction in $4 k$ dimensions. Commun. Math. Phys. 100, 1-13 (1985)

12. Devchand, C.: Oxidation of self-duality to 12 dimensions and beyond. Commun. Math. Phys. 329, 461482 (2014)

13. Devchand, C., Lechtenfeld, O.: Extended self-dual Yang-Mills from the $N=2$ string. Nucl. Phys. B 516, 255-272 (1998) 
14. Devchand, C., Leznov, A.N.: Bäcklund transformation for supersymmetric self-dual theories for semisimple gauge groups and a hierarchy of $A_{1}$ solutions. Commun. Math. Phys. 160, 551-561 (1994)

15. Devchand, C., Ogievetsky, V.: Interacting fields of arbitrary spin and $N>4$ supersymmetric self-dual Yang-Mills equations. Nucl. Phys. B 481, 188-214 (1996)

16. Devchand, C., Spiro, A.: On pseudo-hyperkähler prepotentials. J. Math. Phys. 57, 102501-102537 (2016)

17. Donaldson, S.K.: Anti self-dual Yang-Mills connections over complex algebraic surfaces and stable vector bundles. Proc. Lond. Math. Soc. 50, 1-26 (1985)

18. Donaldson, S.K., Kronheimer, P.B.: The Geometry of Four-Manifolds. The Clarendon Press, New York (1990)

19. Donaldson, S.K., Thomas, R.P.: Gauge theory in higher dimensions. In: The Geometric Universe (Oxford. 1996). pp. 31-47. Oxford Univ. Press, Oxford (1998)

20. Drinfeld, V.G., Manin, Y.I.: A description of instantons. Commun. Math. Phys. 63, 177-192 (1978)

21. Freed, D.S., Uhlenbeck, K.: Instantons and Four-Manifolds. Springer, New York (1991)

22. Galperin, A., Ivanov, E., Ogievetsky, V., Sokatchev, E.: Gauge field geometry from complex and harmonic analyticities. I. Kähler and self-dual Yang-Mills cases. Ann. Phys. 185, 1-21 (1988)

23. Galperin, A., Ivanov, E., Ogievetsky, O.: Harmonic space and quaternionic manifolds. Ann. Phys. 230, 201-249 (1994)

24. Galperin, A.S., Ivanov, E.A., Ogievetsky, V.I., Sokatchev, E.S.: Harmonic Superspace. Cambridge University Press, Cambridge (2004)

25. Harvey, R., Blaine Lawson Jr., H.: Calibrated geometries. Acta Math. 148, 47-157 (1982)

26. LeBrun, C.: Quaternionic-Kähler manifolds and conformal geometry. Math. Ann. 284, 353-376 (1989)

27. Leznov, A.N.: On the equivalence of four-dimensional self-duality equations to a continuous analogue of the problem of a principal chiral field. Teoret. Mat. Fiz. 73, 302-307 (1987). (English translation: Theoret. and Math. Phys. 73 (1987), 1233-1236)

28. Mamone Capria, M., Salamon, S.M.: Yang-Mills fields on quaternionic spaces. Nonlinearity 1, 517-530 (1988)

29. Morgan, J.W.: An introduction to gauge theory. In: Gauge Theory and the Topology of Four-Manifolds (Park City, UT, 1994). pp. 51-143. Amer. Math. Soc., Providence, RI (1998)

30. Nakajima, H.: Compactness of the moduli space of Yang-Mills connections in higher dimensions. J. Math. Soc. Jpn. 40, 383-392 (1988)

31. Nitta, T.: Vector bundles over quaternionic Kähler manifolds. Tohoku Math. J. 40, 425-440 (1988)

32. Palais, R.: A global formulation of the Lie theory of transformation groups. Mem. Am. Math. Soc. No. 22, iii+123 (1957)

33. Polyakov, A.M.: Quark confinement and topology of gauge groups. Nucl. Phys. B 120, 429-458 (1977)

34. Salamon, S.M.: Quaternionic Kähler manifolds. Invent. Math. 67, 143-171 (1982)

35. Siegel, W.: $N=2$ (4) string theory is self-dual $N=4$ Yang-Mills theory. Phys. Rev. D (3) 46, R3235R3238 (1992)

36. Tian, G.: Gauge theory and calibrated geometry, I. Ann. Math. 151, 193-208 (2000)

37. Uhlenbeck, K.K.: Removable singularities in Yang-Mills fields. Commun. Math. Phys. 83, 11-29 (1982)

38. Uhlenbeck, K.K.: Connections with $L^{p}$ bounds on curvature. Commun. Math. Phys. 83, 31-42 (1982)

39. Uhlenbeck, K.K., Yau, S.T.: On the existence of Hermitian-Yang-Mills connections in stable vector bundles. Commun. Pure Appl. Math. 39, S257-S293 (1986)

40. Ward, R.S.: On self-dual gauge fields. Phys. Lett. A 61, 81-82 (1977)

41. Ward, R.S.: Completely solvable gauge-field equations in dimension greater than four. Nucl. Phys. B 236, 381-396 (1984)

42. Wehrheim, K.: Uhlenbeck Compactness. European Mathematical Society, Zürick (2004)

43. Zhang, X.: A compactness theorem for Yang-Mills connections. Can. Math. Bull. 47, 624-634 (2004)

Publisher's Note Springer Nature remains neutral with regard to jurisdictional claims in published maps and institutional affiliations. 